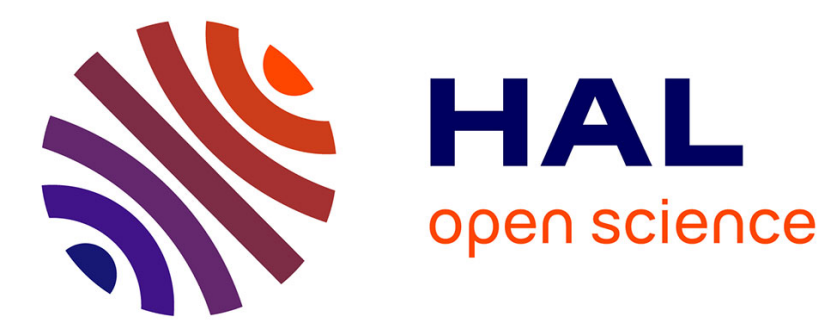

\title{
Chattering-Free Digital Sliding-Mode Control with State Observer and Disturbance Rejection
}

\author{
Vincent Acary, Bernard Brogliato, Yuri V Orlov
}

\section{To cite this version:}

Vincent Acary, Bernard Brogliato, Yuri V Orlov. Chattering-Free Digital Sliding-Mode Control with State Observer and Disturbance Rejection. IEEE Transactions on Automatic Control, 2012, 57 (5), pp.1087-1101. 10.1109/TAC.2011.2174676 . inria-00580713

\section{HAL Id: inria-00580713 \\ https://hal.inria.fr/inria-00580713}

Submitted on 29 Oct 2017

HAL is a multi-disciplinary open access archive for the deposit and dissemination of scientific research documents, whether they are published or not. The documents may come from teaching and research institutions in France or abroad, or from public or private research centers.
L'archive ouverte pluridisciplinaire HAL, est destinée au dépôt et à la diffusion de documents scientifiques de niveau recherche, publiés ou non, émanant des établissements d'enseignement et de recherche français ou étrangers, des laboratoires publics ou privés. 


\title{
Chattering-Free Digital Sliding-Mode Control With State Observer and Disturbance Rejection
}

\author{
Vincent Acary, Bernard Brogliato, and Yury V. Orlov
}

\begin{abstract}
In this paper, a novel discrete-time implementation of sliding-mode control systems is proposed, which fully exploits the multivaluedness of the dynamics on the sliding surface. It is shown to guarantee a smooth stabilization on the discrete sliding surface in the disturbance-free case, hence avoiding the chattering effects due to the time-discretization. In addition, when a disturbance acts on the system, the controller attenuates the disturbance effects on the sliding surface by a factor $h$ (where $h$ is the sampling period). Most importantly, this holds even for large $h$. The controller is based on an implicit Euler method and is very easy to implement with projections on the interval $[-1,1]$ (or as the solution of a quadratic program). The zero-order-hold ( $\mathrm{ZOH})$ method is also investigated. First- and second-order perturbed systems (with a disturbance satisfying the matching condition) without and with dynamical disturbance compensation are analyzed, with classical and twisting sliding-mode controllers.
\end{abstract}

Index Terms-Backward Euler method, discrete-time sliding mode, disturbance compensation, sliding-mode, twisting controller, zero-order-hold method.

\section{INTRODUCTION}

$\mathbf{S}$ LIDING-MODE control is an important field of feedback control, with many applications, see, e.g., [8], [18], [24], [27], [34], and [35]. The issue related to the digital definition and implementation of sliding mode systems, has been the object of many works since the publication of pioneering works [12], [25], see, e.g., [5], [15], [20], [29], [30], [34], [35], and [38]. It appears however that such control methods are not yet fully understood and their implementation is still prone to serious problems like numerical chattering [6], [16], [17], [19], [21], [35]-[37], [39]. The objective of this paper is threefold: 1) to show that an implicit Euler controller permits to numerically implement the multivalued part of discontinuous sliding-mode controllers and consequently suppress the numerical chattering that is present in the explicit implementations [16], [17], [37], 2) to extend it to the case when one part of the state is observed, 3) to show that when a disturbance acts on the system

The work of Y. V. Orlov was supported by Consejo Nacional de Ciencia y Tecnología de México during his sabbatical stay at the University of Kent. Recommended by Associate Editor A. Ferrara.

V. Acary and B. Brogliato are with the INRIA Grenoble Rhône-Alpes, BIPOP project-team, 38334 Saint-Ismier, France (e-mail: vincent.acary@inrialpes.fr; bernard.brogliato@inrialpes.fr).

Y. V. Orlov is with the CICESE, Departamento de Electronica y Telecomunicaciones, Carretera Tijuana-Ensenada 22860, Mexico (e-mail: yorlov@ cicese.mx). (full-state or partial-state feedback) the numerical chattering is still suppressed and the disturbance is rejected. The numerical chattering corresponds to the oscillations (limit cycles) which are solely due to the digital implementation of the controller. The disturbance chattering corresponds to the oscillations that can appear due to a high frequency disturbance acting on the system. By disturbance rejection it is meant that in the ideal (analytical) continuous-time system, the disturbance is exactly rejected, while in the digital implementation it is attenuated by a factor $h$ where $h>0$ is the sampling time. The major features of the implicit causal discrete-time input are on one hand that the continuous-time system sliding surface (that may be of codimension larger than one) is not changed after the discretization, on the other hand a finite sampling frequency is sufficient to assure the sliding motion of the discrete-time system, and finally the chattering effects observed on the closed-loop state with explicit controllers (named the numerical chattering) are suppressed.

A first fundamental step is to eliminate the numerical chattering with the application of a suitable implicit discrete-time controller. The disturbance chattering will not be eliminated in the system's state around the sliding surface, but the disturbance is attenuated by a factor $h$ (of a factor $h^{2}$ on the system's position for an order-two system), which is in accordance with the estimations provided in [22], [23], [32]. In practice it is expected that this corresponds to a high compensation of the disturbance. The control input obtained by the implicit method is not of the bang-bang type when the state evolves on the sliding surface. On the contrary it is a continuous input which evolves inside the multivalued part of the sign multifunction (the multivalued part corresponds in the Filippov case to the set representing the closed convex closure of the vector fields on the switching surface, which is a segment if the codimension is equal to one).

Definition 1: Let $h=t_{k+1}-t_{k}>0$ be the sampling period, $k \geqslant 0$. An $m$-discrete-time sliding surface $\Sigma_{d}$ is a codimension $m$ subspace of the state space, such that the discrete state vector $x_{k} \approx x\left(t_{k}\right)$ satisfies $x_{k} \in \Sigma_{d}$ for all $k_{\min } \leqslant k \leqslant k_{\max }$, $k_{\text {min }}<k_{\text {max }}-1, k_{\text {min }} \geqslant 0$, and all $h>0$.

A very attractive feature of the digital method based on the implicit Euler method is that the numerical sliding surface $\Sigma_{d}$ and the continuous-time sliding surface $\Sigma_{c}$ satisfy $\Sigma_{d} \subset \Sigma_{c}$ : the discretization does not modify the sliding surface [1]. If, for instance, $\Sigma_{c}=\left\{x \in \mathbb{R}^{n} \mid C x+D=0\right\}, C \in \mathbb{R}^{m \times n}, D \in$ $\mathbb{R}^{m}$, then $\Sigma_{d}=\left\{x_{k} \in \mathbb{R}^{n} \mid C x_{k}+D=0\right\}$. The controllers which are designed in this paper consist of the stabilization of an unperturbed nominal plant, coupled to the plant's dynamics. The idea of keeping exact sliding mode in the discrete case is 
not new [12]; however, the systematic design of controllers that guarantee it seems to be novel (see remark 1 below for details).

The paper is organized as follows. Section II is dedicated to the analysis of a simple first-order system, without and with disturbance compensation. An extension to higher-order systems is also presented, with the Euler and the $\mathrm{ZOH}$ methods. In Section III second-order systems are treated and several types of controllers are analyzed. In all cases the continuous-time system is introduced, then its time-discretization is studied, and finally simulation results are shown. Conclusions end the paper.

Notation: In the sequel $\operatorname{sgn}(x)$ is the multivalued sign function:

$$
\operatorname{sgn}(x)= \begin{cases}\{+1\}, & \text { if } x>0 \\ \{-1\}, & \text { if } x>0 \\ {[-1,1],} & \text { if } x=0\end{cases}
$$

where $\{\cdot\}$ is a singleton. Let $K \subset \mathbb{R}^{n}$ be a closed non-empty convex set. The normal cone to $K$ at $x \in K \subset \mathbb{I R}^{n}$ is $N_{K}(x)=$ $\left\{z \in \mathbb{R} R^{n} \mid z^{T}(y-x) \leqslant 0\right.$ for all $\left.y \in K\right\}$. Let $M=M^{T}>0$ be an $n \times n$ positive definite matrix. For any $x \in \mathbb{R}^{n}$ and $y \in \mathbb{R}^{n}$, one has

$$
\begin{aligned}
& -x+y \in M^{-1} N_{K}(x) \\
& \Leftrightarrow x=\operatorname{proj}_{M}(K ; y) \\
& \Leftrightarrow x=\arg \min _{z \in K} \frac{1}{2}(z-y)^{T} M(z-y)
\end{aligned}
$$

where $\operatorname{proj}_{M}(K ; y)$ denotes the orthogonal projection of $y$ on $K$ in the metric defined by $M$. For any reals $x$ and $y$, one has

$$
x \in \operatorname{sgn}(y) \Leftrightarrow y \in N_{[-1,1]}(x) .
$$

Readers not familiar with set-valued functions may have a look at [2, Fig. 1.9] or at [3, Fig. 2.11] for a simple illustration of (2). For $x \in \mathbb{R}^{m}, \operatorname{Sgn}(x)=\left(\operatorname{sgn}\left(x^{1}\right), \ldots, \operatorname{sgn}\left(x^{m}\right)\right)^{T}$, $\|x\|_{\infty}=\max \left(\left|x^{1}\right|, \ldots,\left|x^{m}\right|\right),\|x\|_{1}=\sum_{i=1}^{m}\left|x^{i}\right|$. For any matrix $M$ and vector $x$, the norms $\|M\|$ and $\|x\|$ are supposed to be compatible norms so that $\|M x\| \leqslant\|M\|\|x\|$. For a function $f$ : $I R \rightarrow \mathbb{R}$ one has $\|f\|_{\infty}=\operatorname{ess}_{\sup } \operatorname{suR}_{t \in \mathbb{R}}|f(t)|=\inf \{c|| f(t) \mid \leqslant$ $c$ almost everywhere on $\mathbb{R}\} . I_{n}$ is the $n \times n$ identity matrix. The approximation of the value of a function $f(\cdot)$ at the time $t_{k}$ is denoted as $f_{k}$. The power set of $\mathbb{I R}$, the set of all subsets of $\mathbb{R}$, is denoted by $2^{\mathbb{R}}$. A control input is said causal if it does not explicitly depend on future values of the state or other variables.

\section{FIRST-ORDER SYSTEM}

We analyze in this section the simplest case to illustrate how the method works. Two cases are treated: without and with disturbance compensation (in the continuous-time system). The basic ideas are illustrated on a simple first-order system.

\section{A. The Case Without Disturbance Compensation}

Let us start by considering the following basic sliding mode system:

$$
\left\{\begin{array}{l}
\dot{x}(t)=-a \tau(t)+\varphi(t) \\
\tau(t) \in \operatorname{sgn}(x(t))
\end{array}\right.
$$

where $\varphi(\cdot)$ is the Lebesgue measurable perturbation such that $\|\varphi\|_{\infty}<\rho<a$. The control input is here $u(t)=\tau(t)$. It may be seen, in the language of differential inclusions theory, as a Lebesgue measurable selection of the set-valued right-hand side of the system [33]. Choosing correctly this selection is the object of the following discretization. The system (3) has $x=0$ as its unique equilibrium point, which is globally asymptotically stable and is reached in finite time (this may be shown with the Lyapunov function $V(x)=x^{2}$ ). The discrete-time sliding mode system is implemented as follows:

$$
\left\{\begin{array}{l}
\tilde{x}_{k+1}=x_{k}-a h \tau_{k+1} \\
\tau_{k+1} \in \operatorname{sgn}\left(\tilde{x}_{k+1}\right) \\
x_{k+1}=x_{k}-a h \tau_{k+1}+h \varphi_{k+1} .
\end{array}\right.
$$

The first two lines of (4) may be considered as the nominal unperturbed plant, from which one computes the input at time $t_{k}$. The input is said implicit since it involves $\tilde{x}_{k+1}$ in the sign multifunction. It is however a causal input as shown next, and $\tilde{x}_{k+1}$ is just an intermediate variable which does not explicitly enter into the controller. The third line is the Euler approximation of the plant, on which the disturbance is acting. One has $u(t)=\tau_{k+1}$ on the time-interval $\left[t_{k}, t_{k+1}\right)$.

Proposition 1: Let $x_{0}$ be the given initial state. Then after a finite number of steps $k_{0}$ one obtains that $\tilde{x}_{k}=0$ and $x_{k}=h \varphi_{k}$ for all $k>k_{0}$. In other words, the disturbance is attenuated by a factor $h$. Moreover the approximated derivative of the state satisfies $\left(x_{k+1}-x_{k}\right) / h=\varphi_{k+1}-\varphi_{k}$ for all $k>k_{0}+1$ whereas $\left(\tilde{x}_{k+1}-\tilde{x}_{k}\right) / h=0$ for all $k>k_{0}$. The control input takes values inside the sign multifunction multivalued part on the sliding surface for all $k>k_{0}$.

Proof: Let us start with the case $\left|x_{0}\right|>a h>0$. The generalized equation $\tilde{x}_{k+1}=x_{k}-a h \tau_{k+1}$ and $\tau_{k+1} \in \operatorname{sgn}\left(\tilde{x}_{k+1}\right)$ is found to be equivalent, using (1) and (2), to the inclusion $\tau_{k+1}-$ $\left(x_{k} / a h\right) \in-N_{[-1,1]}\left(\tau_{k+1}\right)$ which is equivalent to $\tau_{k+1}=$ $\operatorname{proj}\left([-1,1] ;\left(x_{k} / a h\right)\right)$. Thus, one obtains the following:

- If $x_{k}>a h$ then $\tilde{x}_{k+1}=x_{k}-a h$ and $\operatorname{sgn}\left(\tilde{x}_{k+1}\right)=1$.

- If $x_{k}<-a h$ then $\tilde{x}_{k+1}=x_{k}+a h$ and $\operatorname{sgn}\left(\tilde{x}_{k+1}\right)=-1$.

- If $0>x_{k}>-a h$ then $\tilde{x}_{k+1} \in(-a h, 0)$, and $\operatorname{sgn}\left(\tilde{x}_{k+1}\right)=-1$.

- If $0<x_{k}<a h$ then $\tilde{x}_{k+1} \in(0, a h)$, and $\operatorname{sgn}\left(\tilde{x}_{k+1}\right)=1$. From the above we infer the following:

- If $x_{k}>a h$ then $x_{k+1}=x_{k}+h \varphi_{k+1}-a h=x_{k}+$ $h\left(\varphi_{k+1}-a\right)<x_{k}+h(\rho-a)$. Since $\rho-a<0$ the state is strictly decreased from step $k$ to step $k+1$.

- If $x_{k}<-a h$ then $x_{k+1}=x_{k}+h \varphi_{k+1}+a h=x_{k}+$ $h\left(\varphi_{k+1}+a\right)>x_{k}+h(a-\rho)$. Since $a-\rho>0$ the state is strictly increased from step $k$ to step $k+1$.

One deduces that if the initial data satisfies $\left|x_{0}\right|>a h$ then after $k_{0}=\left\lceil x_{0} /(h|a-\rho|)\right\rceil$ steps one gets $\tilde{x}_{k_{0}}=0$, where $\lceil x\rceil$ stands for the integer part of $x \in \mathbb{R}$. Indeed at $k_{0}$ the state $x_{k}$ reaches the interval $(-a h, a h)$ and then the unique solution for $\tilde{x}_{k}$ is zero. From $\tilde{x}_{k_{0}}=0$ one deduces that $\left|x_{k_{0}}\right|<a h$. In the case that $\left|x_{0}\right| \leqslant a h$, it is easily to check that $k_{0}=1$.

To compute the next value of $\tilde{x}_{k}$ one has to solve the generalized equation

$$
\left\{\begin{array}{l}
\tilde{x}_{k_{0}+1}=x_{k_{0}}-a h \tau_{k_{0}+1} \\
\tau_{k_{0}+1} \in \operatorname{sgn}\left(\tilde{x}_{k_{0}+1}\right)
\end{array}\right.
$$


whose unique solution is found by inspection to be $\tilde{x}_{k_{0}+1}=0 .{ }^{1}$ The reasoning can be repeated to conclude that $\tilde{x}_{k}=0$ for all $k \geqslant k_{0}$. Therefore, $\left(\tilde{x}_{k+1}-\tilde{x}_{k}\right) / h=0$ for all $k>k_{0}$. Now let us assume that for $k \geqslant k_{0}$ we have

$$
\tilde{x}_{k+1}=x_{k}-a h \tau_{k+1}=0, \quad k \geqslant k_{0}
$$

that is

$$
\tau_{k+1}=\frac{x_{k}}{h a}
$$

In this case, the state $x_{k+1}$ is given by

$$
x_{k+1}=h \varphi_{k+1}
$$

and therefore

$$
x_{k}=h \varphi_{k}, \quad \tau_{k+1}=\frac{\varphi_{k}}{a} \quad \text { for all } \quad k \geq k_{0}+1
$$

so that $\left(x_{k+1}-x_{k}\right) / h=\varphi_{k+1}-\varphi_{k}$ for all $k>k_{0}+1$.

Notice that the backward (or implicit) Euler discretization of the unperturbed plant coincides for (3) with the zero-order holder $(\mathrm{ZOH})$ discretization. Considering the perturbed plant, the only difference between (4) and the $\mathrm{ZOH}$ discretization is that $h \varphi_{k+1}$ becomes $\int_{\left[t_{k}, t_{k+1}\right)} \varphi(t) d t$, and $h \varphi_{k+1}$ in (8) and (9)

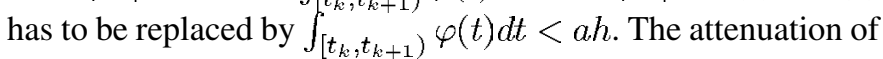
the disturbance still holds with the $\mathrm{ZOH}$ method. In other words, the state $x(\cdot)$ of the plant satisfies $x(t)=\int_{\left[t_{k}, t\right)} \varphi(t) d t \leqslant h \rho$. In a more general setting, the discretization of the controller and the discretization of the plant have to be the same (both implicit Euler, or both $\mathrm{ZOH}$ ) in order for the disturbance attenuation to hold. Notice that the above shows that $V_{k}=\left|\tilde{x}_{k}\right|$ is a Lyapunov function for the nominal system.

\section{B. The Case With Disturbance Compensation}

Let us consider the case with disturbance compensation. For the purpose of compensating a disturbance affecting the underlying system, let us define the compensator variable $\hat{x}$ through the dynamic equation $\dot{\hat{x}}(t)=-a \tau_{1}(t), \tau_{1}(t) \in \operatorname{sgn}(x(t))$, $e=x-\hat{x}$, and the controller $u=-\operatorname{asgn}(x(t))-\alpha \operatorname{sgn}(e(t))$, $a>0, \alpha>0$ and $a<\alpha$. Thus, the closed-loop system is given by

$$
\left\{\begin{array}{l}
\dot{x}(t)=-a \tau_{1}(t)-\alpha \tau_{2}(t)+\varphi(t) \\
\dot{e}(t)=-\alpha \tau_{2}(t)+\varphi(t) \\
\tau_{1}(t) \in \operatorname{sgn}(x(t)) \\
\tau_{2}(t) \in \operatorname{sgn}(e(t))
\end{array}\right.
$$

where $\varphi(\cdot)$ is a disturbance such that $\|\varphi\|_{\infty}<\rho<\min (a, \alpha)$. The fixed point $(x, e)=(0,0)$ of the system may be shown in a rather standard way [34] to be globally strongly asymptotically stable with the nonsmooth Lyapunov function $V(x, e)=$ $|x|+|e|$. Moreover, the system attains in a finite time the sliding surface $e=0$ where it evolves according to the sliding dynamics $\dot{x}(t)=-a \tau_{1}(t)+\varphi(t)$. The condition $a<\alpha$ implies that the

\footnotetext{
${ }^{1}$ The underlying crucial property that makes this hold is the maximal monotonicity of the sign multifunction.
}

origin is not attained directly, but first the system slides on the surface $e=0$. On this surface it is apparent from (10) that the dynamics in $x$ evolves as a disturbance-free system. The discrete sliding mode system is implemented as follows:

$$
\left\{\begin{array}{l}
\tilde{x}_{k+1}=x_{k}-a h \tau_{1, k+1}-\alpha h \tau_{2, k+1} \\
\tilde{e}_{k+1}=e_{k}-\alpha h \tau_{2, k+1} \\
\tau_{1, k+1} \in \operatorname{sgn}\left(\tilde{x}_{k+1}\right) \\
\tau_{2, k+1} \in \operatorname{sgn}\left(\tilde{e}_{k+1}\right)
\end{array}\right.
$$

and the update procedure representing the plant dynamics is given by

$$
\left\{\begin{array}{l}
x_{k+1}=x_{k}-a h \tau_{1, k+1}-\alpha h \tau_{2, k+1}+h \varphi_{k+1} \\
e_{k+1}=e_{k}-\alpha h \tau_{2, k+1}+h \varphi_{k+1} .
\end{array}\right.
$$

Proposition 2: Let $x_{0}, e_{0}$ be the initial conditions of (11). Then after a finite number of steps $k_{0}$ one obtains $\tilde{e}_{k}=0$ and $e_{k}=h \varphi_{k+1}$ for all $k>k_{0}$. There exists $k_{1}<+\infty$ such that $\tilde{x}_{k}=0$ for all $k>k_{0}+k_{1}$ and $x_{k}=h \varphi_{k}$ for all $k \geqslant k_{0}+k_{1}$.

The proof is in Appendix A. Consequently, the discrete-time controller guarantees the convergence of the state of the nominal system in finite time to the origin, while the plant's state is equal to the disturbance attenuated by a factor $h$. To summarize, from (11) and (12) the discrete-time closed-loop system is therefore

$$
\left\{\begin{array}{l}
x_{k+1}=x_{k}-a h \tau_{1, k+1}-\alpha h \tau_{2, k+1}+h \varphi_{k+1} \\
e_{k+1}=e_{k}-\alpha h \tau_{2, k+1}+h \varphi_{k+1} \\
\tau_{1, k+1}=\operatorname{proj}\left([-1,1] ; \frac{x_{k}-\alpha h \tau_{2, k+1}}{a h}\right) \\
\tau_{2, k+1}=\operatorname{proj}\left([-1,1] ; \frac{e_{k}}{\alpha h}\right) .
\end{array}\right.
$$

One sees that this is very easily implementable with nested projections.

\section{Extension to Higher Order Systems}

In order to show that the foregoing method extends to $n$ th-order systems with the equivalent-control-based sliding-mode-controller (ECB-SMC [35, Ch. 2]) and also to better fix the ideas on the structure of the proposed controllers, let us consider the linear time-invariant system with disturbance $\dot{x}(t)=A x(t)+B u(t)+D \varphi(t)$ with $\|\varphi(t)\|_{1} \leqslant p \varphi_{\max }$ for all $t$, $\varphi_{\max } \geqslant\left|\varphi^{i}\right|_{\infty}$ for all $1 \leqslant i \leqslant p$ and $D \in \mathbb{R}^{n \times p}$. Let us choose a sliding surface $\Sigma=\left\{x \in \mathbb{R}^{n} \mid C x=0, C \in \mathbb{R}^{m \times n}\right\}$, where $m$ is the dimension of the input vector $u(t)$. The ECB-SMC takes the form $u \in-(C B)^{-1} C A x-\alpha(C B)^{-1} \operatorname{Sgn}(C x)$, provided $C B$ is full-rank. Let $z \triangleq C x$. The reduced closed-loop dynamics is $\dot{z}(t)=-\alpha \tau+C D \varphi(t), \tau \in \operatorname{Sgn}(z)$, which is globally asymptotically stable and $\Sigma$ is reached in finite time provided $\alpha>p\|C D\| \varphi_{\max }$ (this can be shown with the Lyapunov function $V(z)=(1 / 2) z^{T} z$ that satisfies along the closed-loop trajectories $\left.\dot{V}(t) \leqslant\|z\|_{1}\left(-\alpha+p\|C D\| \varphi_{\max }\right)\right)$. The system is discretized as

$$
x_{k+1}=x_{k}+h A x_{k}+h B u_{k+1}+h D \varphi_{k+1}
$$


and the nominal system is simply given by $\tilde{x}_{k+1}=$ $(I+h A) x_{k}+h B u_{k+1}$. The implicit Euler controller is defined as

$$
\left\{\begin{array}{l}
u_{k+1}=-(C B)^{-1} C A x_{k}-\alpha(C B)^{-1} \tau_{k+1} \\
\tau_{k+1} \in \operatorname{Sgn}\left(C \tilde{x}_{k+1}\right)
\end{array}\right.
$$

Therefore, $\tau_{k+1}$ is given by [see (1) and (2)]

$$
\begin{aligned}
\tau_{k+1} & \in \operatorname{Sgn}\left(C x_{k}-\alpha h \tau_{k+1}\right) \\
\Leftrightarrow \tau_{k+1} & =\operatorname{proj}\left([-1,1]^{m} ; \frac{1}{\alpha h} C x_{k}\right)
\end{aligned}
$$

where $[-1,1]^{m}=[-1,1] \times \ldots \times[-1,1] m$-times. Thus, the controller to be applied at time $t_{k}$ is

$u_{k+1}=-(C B)^{-1} C A x_{k}-\alpha(C B)^{-1} \operatorname{proj}\left([-1,1]^{m} ; \frac{1}{\alpha h} C x_{k}\right)$.

We therefore obtain, with $z_{k}=C x_{k}$ and $\tilde{z}_{k}=C \tilde{x}_{k}$ :

$$
\left\{\begin{array}{l}
\tilde{z}_{k+1}=z_{k}-\alpha h \tau_{k+1} \\
\tau_{k+1} \in \operatorname{Sgn}\left(\tilde{z}_{k+1}\right) \\
z_{k+1}=z_{k}-\alpha h \tau_{k+1}+h C D \varphi_{k+1}
\end{array}\right.
$$

that is similar to (4). Thus, the same conclusions as in Proposition 1 may be drawn for this discrete-time system provided that $\alpha>p\|C D\| \varphi_{\max }$ : the sliding surface $C \tilde{x}_{k}=0$ is attained after a finite-number of steps whatever the bounded initial state, and the discrete-time system evolves smoothly on this surface while the disturbance effects on the variable $C x_{k}$ are attenuated by a factor $h$.

Remark 1: The discrete-time input obtained from [35, Eq.(9.36)] (see also [5], [20] and [12] for the original contribution) when applied to (14) is calculated to be: $u_{k+1}=-(h C B)^{-1} C(I+h A) x_{k}=-(C B)^{-1}\left(C x_{k} / h\right)-$ $(C B)^{-1} C A x_{k}$, which is linear. The discrepancy with (17) is the projection on the set $[-1,1]^{m}$ that is intrinsically present in the implicit Euler input (that is nonlinear Lipschitz continuous), and is not a consequence of adding saturations because of actuator limitations. Also the controller in (17) remains bounded when $h \rightarrow 0$, a property shared by all the controllers considered in this paper. One may say that both controller designs share the same "philosophy" since they are both calculated in order to force the discrete sliding surface to be zero, with a suitable input. However, they are not at all equivalent. In practice, the controllers proposed in this paper may be calculated using a suitable complementarity problem solver [2].

As alluded to in Section II-A, the plant and the controller have to be discretized with the same method (backward Euler or $\mathrm{ZOH}$ ) in order to assure the disturbance attenuation. Let us investigate the zero-order-holder method $(\mathrm{ZOH})$ on this example. The input is assumed to be constant on $\left[t_{k}, t_{k+1}\right]$ and is computed at $t=t_{k}$. The ZOH discretization of the ECB-SMC controller on $\left[t_{k}, t_{k+1}\right]$ takes the form [39]

$$
x_{k+1}=A^{*}(h) x_{k}-\alpha B^{*}(h) \tau_{k+1}+\varphi^{*}(h)
$$

with $A^{*}(h)=e^{A h}-\int_{0}^{h} e^{A t} d t B(C B)^{-1} C A, B^{*}(h)=$ $\int_{0}^{h} e^{A t} d t B(C B)^{-1}, \varphi_{k}^{*}(h)=\int_{0}^{h} e^{A t} D \varphi((k+1) h-\tau) d \tau$.
Notice that as $h \rightarrow 0$ then $A^{*}(h) \approx I_{n}+A h-h B(C B)^{-1} C A+$ $\mathcal{O}\left(h^{2}\right), B^{*}(h) \approx h B(C B)^{-1}+\mathcal{O}\left(h^{2}\right)$, consequently the implicit Euler and $\mathrm{ZOH}$ methods yield the same discrete-time system when the sampling period is small. Also one may compute that $\left\|\varphi_{k}^{*}(h)\right\|_{1} \leqslant h p\|D\| \varphi_{\max }+\mathcal{O}\left(h^{2}\right)$. This yields the generalized equation

$$
\begin{gathered}
\text { (a) }\left\{\begin{array}{l}
\tilde{x}_{k+1}=A^{*}(h) x_{k}-\alpha B^{*}(h) \tau_{k+1} \tau_{k+1} \in \operatorname{Sgn}\left(C \tilde{x}_{k+1}\right) \\
x_{k+1}=A^{*}(h) x_{k}-\alpha B^{*}(h) \tau_{k+1}+C \varphi_{k}^{*}(h)
\end{array}\right. \\
\Rightarrow(b)\left\{\begin{array}{c}
C \tilde{x}_{k+1}=C A^{*}(h) x_{k}-\alpha C B^{*}(h) \tau_{k+1} \tau_{k+1} \\
\in \operatorname{Sgn}\left(C \tilde{x}_{k+1}\right) \\
C x_{k+1}=C A^{*}(h) x_{k}-\alpha C B^{*}(h) \tau_{k+1} \\
+C \varphi_{k}^{*}(h) .
\end{array}\right.
\end{gathered}
$$

Suppose that the matrix $C B^{*}(h)$ is symmetric positive definite (since $C B^{*}=h I_{m}+\mathcal{O}\left(h^{2}\right)$ it follows that for $h$ small enough $C B^{*}>0$ is guaranteed if $C B$ is invertible). Then from (1) and (2) the first two lines of (20) (b) are equivalent to

$$
\begin{aligned}
& C A^{*}(h) x_{k}-\alpha C B^{*}(h) \tau_{k+1} \in N_{[-1,1]^{m}}\left(\tau_{k+1}\right) \\
& \Leftrightarrow \tau_{k+1}=\operatorname{proj}_{C B^{*}(h)}\left([-1,1]^{m} ; \frac{1}{\alpha}\left(C B^{*}(h)\right)^{-1} C A^{*}(h) x_{k}\right) \\
& \Leftrightarrow \tau_{k+1}=\arg \min _{z \in[-1,1]^{m n}} \frac{1}{2}\left(z-\zeta_{k}\right)^{T} C B^{*}(h)\left(z-\zeta_{k}\right)
\end{aligned}
$$

where $\operatorname{proj}_{C B^{*}(h)}$ is the projection in the metric defined by $C B^{*}(h)$, and $\zeta_{k}=(1 / \alpha)\left(C B^{*}(h)\right)^{-1} C A^{*}(h) x_{k}$. Therefore, at each step the controller is calculated as the solution of a quadratic program and is unique. Notice that when $h$ is small then $C B^{*}(h) \approx h I_{m}$ and $C A^{*}(h) \approx C$ so that

$$
\begin{aligned}
\tau_{k+1} & =\arg \min _{z \in[-1,1]^{m}} \frac{1}{2}\left(z-\frac{1}{h \alpha} C x_{k}\right)^{T}\left(z-\frac{1}{h \alpha} C x_{k}\right) \\
\Leftrightarrow \tau_{k+1} & =\operatorname{proj}\left([-1,1]^{m} ; \frac{1}{h \alpha} C x_{k}\right) .
\end{aligned}
$$

The input remains bounded when the sampling time decreases. The next result is obvious from (20) (b):

Lemma 1: Let $C \tilde{x}_{k+1}=0$ for some $k \geqslant 0$. Then $\left\|C x_{k+1}\right\|_{1}=\left\|C \varphi_{k}^{*}(h)\right\| \leqslant h p\|C\|\|D\| \varphi_{\max }$.

Thus, the disturbance attenuation on the nominal discretetime system sliding surface holds with the $\mathrm{ZOH}$ method. If the higher order terms in $h^{2}$ are neglected, one sees that (20) (b) is the same as (18) where only the disturbance term is modified, so that once again the conclusions of Proposition 1 apply: the discrete-time system reaches the nominal system sliding surface in a finite number of steps. The analysis for any $h>0$ is more involved because the terms in $h^{2}$ introduce a coupling between (20) (b) and (a). However, since we are focusing on the sliding modes and finite-time convergence to the sliding surface only, we may assume that the solution $x(\cdot)$ of the closed-loop system is bounded for any bounded initial data, and that the solution $x_{k}$ of its $\mathrm{ZOH}$ counterpart in (20) (a) is bounded as well, i.e., $\left\|x_{k}\right\| \leqslant M$ for all $k \geqslant 0$ and some $M$. Then the following holds:

Proposition 3: Let $h>0$ be given. Suppose that the solution of (20) (a) satisfies $\left\|x_{k}\right\| \leqslant M$ for all $k \geqslant 0$ and some $M<+\infty$, and that $C B^{*}(h)$ is symmetric positive definite, with $C B^{*}(h) \geqslant \gamma I_{m}>0$ for some known $\gamma$. Then there exists 
a constant $\delta\left(h^{2}, M\right)$ such that if $\alpha>(m / \gamma)\|C\| h \rho\|D\| \varphi_{\max }+$ $\delta\left(h^{2}, M\right), C \tilde{x}_{k+1}=0$ for some $k \geqslant 0$ implies $C \tilde{x}_{k+n}=0$ for all $n \geqslant 2$.

Proof: From Lemma 1 the first line of (20) (b) is rewritten at step $k+2$ as

$$
\begin{aligned}
C \tilde{x}_{k+2} & =\left(C+\mathcal{O}\left(h^{2}\right)\right) x_{k+1}-\alpha C B^{*}(h) \tau_{k+2} \\
& =C \varphi_{k}^{*}(h)+\mathcal{O}\left(h^{2}\right) x_{k+1}-\alpha C B^{*}(h) \tau_{k+2}
\end{aligned}
$$

Thus, (23) and $\tau_{k+2} \in \operatorname{Sgn}\left(C \tilde{x}_{k+2}\right)$ form a generalized equation which possesses a unique solution because $\alpha C B^{*}(h)$ is positive definite. We may rewrite it as

$$
\begin{aligned}
0 \in \frac{1}{\alpha}\left(C B^{*}(h)\right)^{-1} C \tilde{x}_{k+2}-\frac{1}{\alpha}\left(C B^{*}(h)\right)^{-1} \\
\quad \times\left(C \varphi_{k}^{*}(h)+\mathcal{O}\left(h^{2}\right) x_{k+1}\right)+\operatorname{Sgn}\left(C \tilde{x}_{k+2}\right) .
\end{aligned}
$$

Therefore, if $(1 / \alpha)\left(C B^{*}(h)\right)^{-1}\left(C \varphi_{k}^{*}(h)+\mathcal{O}\left(h^{2}\right) x_{k+1}\right) \in$ $[-1,1]^{m}$ then $C \tilde{x}_{k+2}=0$ is the unique solution of (24). From the proposition's assumptions one has

$$
\begin{array}{r}
\left\|\left(C B^{*}(h)\right)^{-1}\left(C \varphi_{k}^{*}(h)+\mathcal{O}\left(h^{2}\right) x_{k+1}\right)\right\|_{1} \\
\leqslant \frac{m}{\gamma}\|C\| h p\|D\| \varphi_{\max }+\delta\left(h^{2}, M\right)
\end{array}
$$

where $\delta\left(h^{2}, M\right)$ is an upper bound for $\mathcal{O}\left(h^{2}\right) x_{k+1}$. This upper bound depends only on $M$, the system's matrices, and $h$. It is therefore uniform with respect to the step number $k$.

Then Lemma 1 may be applied to show the disturbance attenuation on the nominal system discrete-time sliding surface.

\section{Numerical Simulations}

The numerical simulations are obtained with the SICONOS software package of the INRIA ${ }^{2}$ that is dedicated to non-smooth dynamical systems. In order to reproduce the continuous-time nature of the plant, the plant dynamics is integrated in all the simulations with the machine precision, whereas the controller sampling time is much larger: $h=10^{-1} \mathrm{~s}$. This is equivalent to implementing a $\mathrm{ZOH}$ method. The disturbance is taken as $\varphi(t)=\phi \sin (\omega t)$ and we simulate the system in (10).

The above developments are illustrated in Fig. 1 with $a=1$, $\alpha=2, \omega=5$ and $\phi=0.1$. Illustrations are given in Figs. 2 and 3 with $a=1, \alpha=2, \omega=100$, and $\phi=0.1$. The disturbance attenuation is clearly shown.

\section{SECOND-ORDER SYSTEMS}

Let us now focus on a more general class of systems and perform the same steps as for the first-order case (a short recall of the continuous-time case, and then the time-discretization). The simulations will be given after the theoretical presentations.

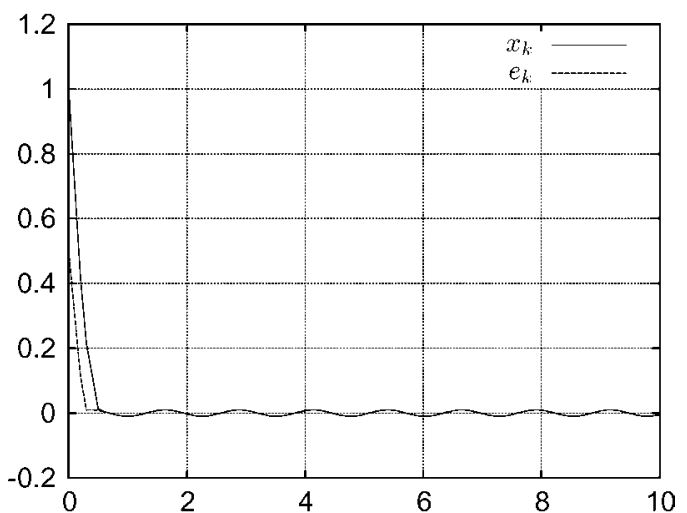

(a)

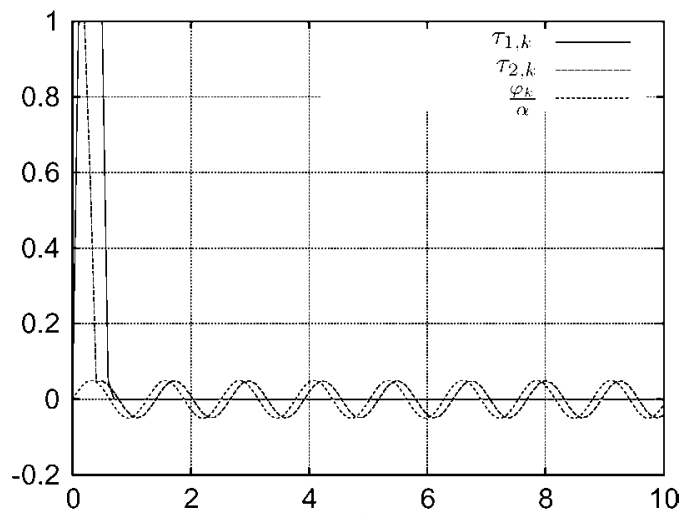

(b)

Fig. 1. Simulation of the system (10), $\phi=0.1, \omega=5$. (a) State $x_{k}$, and error $e_{k}$ versus time; (b) multiplier $\tau_{1, k+1}, \tau_{2, k+1}$ and perturbation $\varphi_{k} / \alpha$ versus time.

\section{A. First-Order Sliding-Mode Stabilization With Disturbance Compensation}

1) The Continuous-Time System: The plant dynamics is given by

$$
\ddot{x}(t)=u(t)+\varphi(x(t), t)
$$

where $x(t) \in \mathbb{R}$ is the state vector, $u(t) \in \mathbb{R}$ is the control input. The disturbance $\varphi(x, t) \in \mathbb{R}$ represents the system uncertainty and its influence on the control process should be rejected. It is assumed that $\varphi(x, t)$ is an unknown function with an a priori known upper estimate $\varphi_{\max }>0$ such that

$$
|\varphi(x, t)|<\varphi_{\max }
$$

for almost all $x, t \in \mathbb{R}$. The model repeats the structure of the plant and is given by

$$
\ddot{\hat{x}}(t)=u(t)+v(t)
$$

where $v(t) \in \mathbb{R}$ is the model input. The error dynamics is then written as follows:

$$
\ddot{e}(t)=-v(t)+\varphi(x(t), t)
$$

where $e=x-\hat{x}$ is the deviation of the model state from the plant state. The error dynamics, driven by the sliding-mode input, is given by

$$
v(t) \in k_{e} \dot{e}(t)+k_{s} s_{e}(t)+M_{v} \operatorname{sgn}\left(s_{e}(t)\right)
$$




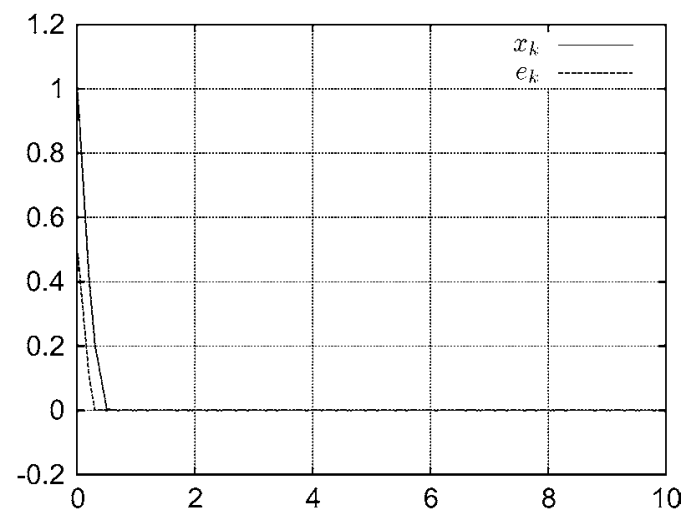

(a)

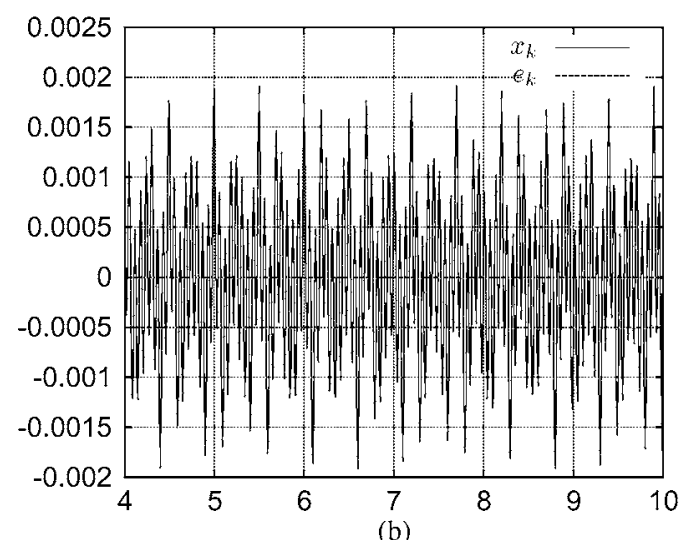

(b)

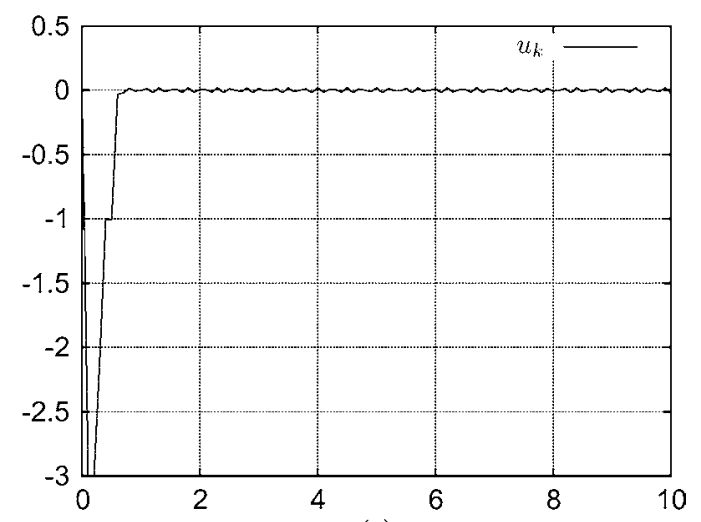

(c)

Fig. 2. Simulation of the system (10) with $\varphi(t)=\phi \sin \omega t, \phi=0.1, \omega=$ 100. (a) State versus time (fine sampling). (b) State versus time (fine sampling, zoom). (c) Control input $u(t)$.

and it is globally asymptotically stabilized provided that $M_{v}>$ $\varphi_{\max }$ and $s_{e}(t)=\dot{e}(t)+k_{e} e(t)$ where $k_{e}$ and $k_{s}$ are positive constants. To reproduce this conclusion it suffices to rewrite the state equation for $s_{e}$, thus arriving at the equation

$$
\dot{s}_{e}(t) \in-k_{s} s_{e}(t)-M_{v} \operatorname{sgn}\left(s_{e}(t)\right)+\varphi(x(t), t)
$$

which has $s_{e}^{*}=0$ as its unique fixed point, which is globally finite-time stable. Thus, by the equivalent control method one has that

$$
v_{e q}(t)=\varphi(x(t), t)
$$

on the surface $s_{e}=0$ and it is expected that the control law

$$
u(t) \in-v(t)-M_{x} \operatorname{sgn}\left(s_{x}(t)\right)-k_{x} \dot{x}(t)
$$

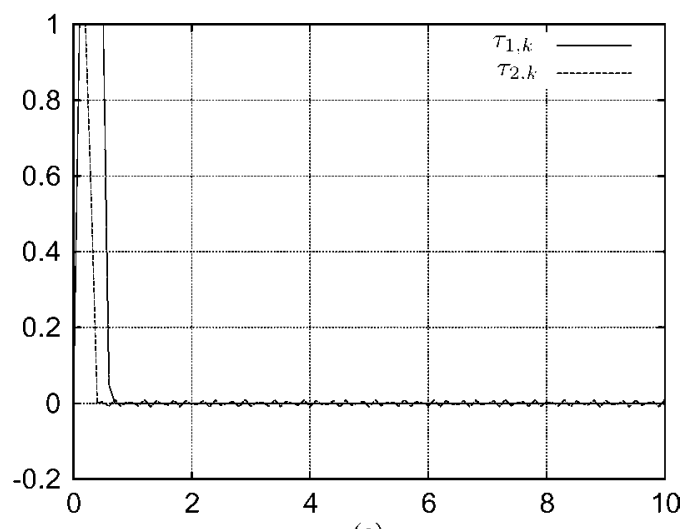

(a)

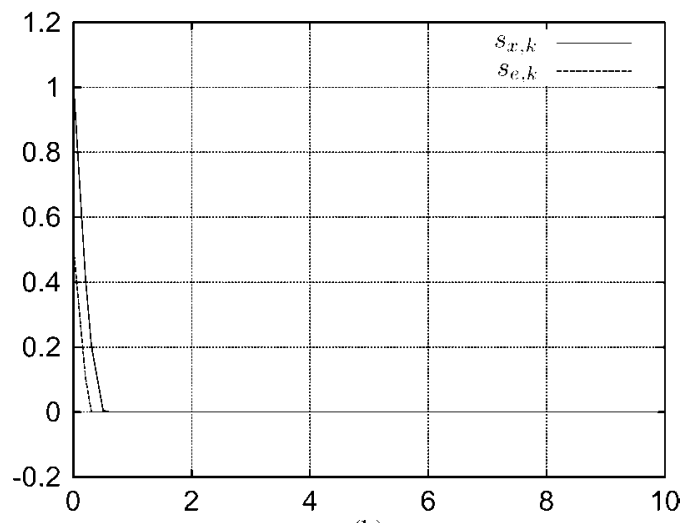

(b)

Fig. 3. Simulation of the system (10) with $\varphi(t)=\phi \sin \omega t, \omega=100$. (a) Multiplier $\tau$. (b) Sliding variable $s(t)$.

with $s_{x}(t)=\dot{x}(t)+k_{x} x(t)$, asymptotically compensates for the disturbance $\varphi(x, t)$. Indeed, once the sliding mode occurs on the surface $s_{e}=0$, the plant equation takes the disturbance-free form

$$
\left\{\begin{array}{l}
\dot{s}_{x}(t) \in-M_{x} \operatorname{sgn}\left(s_{x}(t)\right)-k_{e} \dot{e}(t) \\
\dot{e}(t)=-k_{e} e(t)
\end{array}\right.
$$

because on this sliding surface one has $M_{v} \operatorname{sgn}\left(s_{e}(t)\right)=$ $\varphi(x(t), t)$. Since the dynamics (34) has $s_{x}^{*}=0$ as a globally asymptotically stable fixed point, the desired disturbance compensation is thus provided. Summarizing, the following result, guaranteeing the global asymptotic stability of the closed-loop system, is obtained. Let us denote by $z$ the state

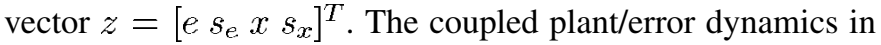
the closed-loop system is given by

$$
\left\{\begin{array}{c}
\dot{z}(t)=\left[\begin{array}{cccc}
-k_{e} & 1 & 0 & 0 \\
0 & -k_{s} & 0 & 0 \\
0 & 0 & -k_{x} & 1 \\
-k_{e} & -k_{s} & 0 & 0
\end{array}\right] z(t) \\
-\left[\begin{array}{cc}
0 & 0 \\
M_{v} & 0 \\
0 & 0 \\
M_{v} & M_{x}
\end{array}\right] \tau(t)+\left[\begin{array}{c}
0 \\
\varphi(x(t), t) \\
0 \\
\varphi(x(t), t)
\end{array}\right] \\
\tau(t) \in \operatorname{Sgn}\left(\left[\begin{array}{llll}
0 & 1 & 0 & 0 \\
0 & 0 & 0 & 1
\end{array}\right] z(t)\right) .
\end{array}\right.
$$

It is noteworthy that the $\left(e s_{e}\right)$ subdynamics is decoupled from the $\left(x s_{x}\right)$ subdynamics without perturbations. 
Proposition 4: Consider the closed-loop system (35) with positive gains $k_{e}, k_{s}, M_{x}, M_{v}$ and an external disturbance $\varphi(x, t)$ such that (27) holds for almost all $x \in \mathbb{R}, t \in \mathbb{R}$ and $M_{v}>\varphi_{\max }$. Then after a finite time, this system evolves in the sliding mode along the surfaces $s_{e}=0$ and $s_{x}=0$, and along these surfaces, the system dynamics is governed by the asymptotically stable, disturbance-free equations (34).

The proof of Proposition 4 is rather standard [34] and it is therefore omitted. The parameter subordination $k_{v} \gg k_{x}$ ensures a faster convergence of the error dynamics compared to the state variables of the plant whereas the controller magnitude $M_{x}$ is required to be positive only. As a matter of fact, the higher $M_{x}$ the higher the plant convergence rate.

2) The Backward Euler Time-Discretization: Let us proceed with the same discretization as in the above first-order examples. For this let us consider the first error dynamics in (31), and discretize it on $\left[t_{k}, t_{k+1}\right)$ as

$$
\left\{\begin{array}{l}
\tilde{s}_{e, k+1}=s_{e, k}-h k_{s} s_{e, k}-h M_{v} \tau_{1, k+1} \\
\tau_{1, k+1} \in \operatorname{sgn}\left(\tilde{s}_{e, k+1}\right) \\
s_{e, k+1}=s_{e, k}-h k_{s} s_{e, k}-h M_{v} \tau_{1, k+1}+h \varphi\left(x_{k+1}, t_{k+1}\right) \\
e_{k+1}=e_{k}+h \dot{e}_{k+1}
\end{array}\right.
$$

for all $k \geqslant 0$. The first two lines are a generalized equation with unknown $\tilde{s}_{e, k+1}$, which we may rewrite as $0 \in F\left(\tilde{s}_{e, k+1}\right)$ for some multifunction $F: \mathbb{R} \rightarrow 2^{\mathbb{R}}$. It has a unique solution since the sign multifunction is maximal monotone and $F(\cdot)$ is 2-monotone as the sum of a monotone and a 2-monotone multifunctions (see Definition 2.3.1 and Theorem 2.3.3 in [13], and Exercise 12.4 in [31]). Notice that if $\tilde{s}_{e, k+1}=0$ then $(1-$ $\left.h k_{s}\right) s_{e, k}=h M_{v} \tau_{1, k+1}$ and $s_{e, k+1}=h \varphi\left(x_{k+1}, t_{k+1}\right)$. Also $\tau_{1, k+1}$ is a function of $s_{e, k}$ only, that is of $\dot{e}_{k}=\left(e_{k}-e_{k-1}\right) / h$ and $e_{k}$. So there is not an exact compensation as in the continuous-time case, but a disturbance-attenuation by a factor $h$. Notice that (36) is exactly (4), by replacing $x_{k}$ with $\left(1-h k_{s}\right) s_{e, k}$, $-a$ with $-M_{v}$. Hence, the conclusions of Proposition 1 hold for (36). We infer that after a finite number of steps $k_{0}$, one obtains $\tilde{s}_{e, k}=0$ and $s_{e, k}=h \varphi\left(x_{k+1}, t_{k+1}\right)$ so that $\left|s_{e, k}\right|<h \varphi_{\max }$ for all $k \geqslant k_{0}$ for some finite $k_{0}$.

The next result characterizes the evolution of $e_{k}$ on the sliding surface $\tilde{s}_{e, k}=0$.

Lemma 2: Suppose that the sliding surface $\tilde{\Sigma}_{e}=\left\{\tilde{s}_{e, k} \in\right.$ $\left.I R \mid \tilde{s}_{e, k}=0\right\}$ is attained at $k=k_{0}$ and that the system stays on it. Take for simplicity $k_{0}=0$. Then

$e_{k+1}=\left(1+h k_{e}\right)^{-k-1} e_{0}+h^{2}\left(1+h k_{e}\right)^{-1} \sum_{i=0}^{k}\left(1+h k_{e}\right)^{i-k} \varphi_{i}$

with $\varphi_{i}=\varphi\left(x_{i}, t_{i}\right)$.

Proof: One has $e_{k}=e_{k-1}+h \dot{e}_{k}$ and $s_{e, k}=\dot{e}_{k}+k_{e} e_{k}$. We infer that

$$
e_{k}=\left(1+h k_{e}\right)^{-1} e_{k-1}+\left(1+h k_{e}\right)^{-1} h^{2} \varphi_{k+1}
$$

from which (37) follows.
Notice that if we implement $e_{k+1}=e_{k}+h \dot{e}_{k}$ then we obtain $e_{k}=\left(1-h k_{e}\right) e_{k-1}+h^{2} \varphi_{k}$ and similar calculations may be done, using the fact that for $h>0$ small enough $0<1-$ $h k_{e}<1$. Therefore, on the sliding surface the discrete-time error is the sum of an asymptotically vanishing term, plus a term that depends on the disturbance, attenuated by a factor $h^{2}$. The second part of the error dynamics in (34) is now discretized as follows:

$$
\left\{\begin{array}{l}
\tilde{s}_{x, k+1}=s_{x, k}-h k_{e} \dot{e}_{k} \\
\quad-h k_{s} s_{e, k}-h M_{v} \tau_{1, k+1}-h M_{x} \tau_{2, k+1} \\
\tau_{2, k+1} \in \operatorname{sgn}\left(\tilde{s}_{x, k+1}\right) \\
s_{x, k+1}=s_{x, k}-h k_{e} \dot{e}_{k}-h k_{s} s_{e, k}-h M_{v} \tau_{1, k+1} \\
\quad-h M_{x} \tau_{2, k+1}+h \varphi\left(x_{k+1}, t_{k+1}\right) \\
\\
x_{k+1}=x_{k}+h \dot{x}_{k+1}
\end{array}\right.
$$

Notice that if $k \geqslant k_{0}$ then $\left(1-h k_{s}\right) s_{e, k}=h M_{v} \tau_{1, k+1}$ and $s_{e, k}=h \varphi\left(x_{k+1}, t_{k+1}\right)$. For $k>k_{0}$ the system evolves on the sliding surface $\tilde{s}_{e, k}=0$ and we obtain

$$
\left\{\begin{array}{l}
\tilde{s}_{x, k+1}=s_{x, k}-h \varphi\left(x_{k+1}, t_{k+1}\right)-h M_{x} \tau_{2, k+1} \\
\tau_{2, k+1} \in \operatorname{sgn}\left(\tilde{s}_{x, k+1}\right) \\
s_{x, k+1}=s_{x, k}-h k_{e} \dot{e}_{k}-h M_{x} \tau_{2, k+1}
\end{array}\right.
$$

From (37) we infer that $h k_{e} \dot{e}_{k}=\epsilon_{k}+h^{2} \alpha_{k}$ where $\left|\alpha_{k}\right| \leqslant$ $\varphi_{0} \sum_{i=0}^{k}\left(1+h k_{e}\right)^{i-k-1}$ and $\epsilon_{k}$ is exponentially decreasing since $1+h k_{e}>1$. It follows also that $\left|\alpha_{k}\right|$ is upper bounded by a constant not depending on $k$ and we may write $\left|\alpha_{k}\right| \leqslant \alpha \varphi_{\max }$ for some constant $\alpha$. We therefore rewrite (40) as

$$
\left\{\begin{array}{l}
\tilde{s}_{x, k+1}=s_{x, k}-h \varphi\left(x_{k+1}, t_{k+1}\right)-h M_{x} \tau_{2, k+1} \\
\tau_{2, k+1} \in \operatorname{sgn}\left(\tilde{s}_{x, k+1}\right) \\
s_{x, k+1}=s_{x, k}-\epsilon_{k}-h^{2} \alpha_{k}-h M_{x} \tau_{2, k+1}
\end{array}\right.
$$

It is noteworthy that (41) is similar to (66) and to (4) except for the exponentially decaying term $\epsilon_{k}$. Thus, the following holds, which shows that the disturbance effects are still attenuated by a factor $h$ :

Proposition 5: Consider the discrete-time system (41) that represents the system's dynamics on the sliding surface $\tilde{\Sigma}_{e}=$ $\left\{\tilde{s}_{e, k} \in \mathbb{I R} \mid \tilde{s}_{e, k}=0\right\}$, i.e., for $k \geqslant k_{0}$. Suppose that $M_{x}>$ $\varphi_{\max }$. There exists $k_{1}<+\infty, k_{1}>k_{0}$, such that for all $k \geqslant k_{1}$ one has $\tilde{s}_{x, k}=0$. Then $\left|s_{x, k+1}\right| \leqslant h \varphi_{\max }+\left|\epsilon_{k}\right|+h^{2} \alpha \varphi_{\max }$.

Proof: The first part of the proof follows the same lines as the above proofs of finite-time convergences and is omitted. The second part follows easily from (41) by imposing $\tilde{s}_{x, k+1}=0$ and inserting the value of $h M_{x} \tau_{2, k+1}$ into the third line of (41).

The next result characterizes the dynamics of $x_{k}$ on the sliding surface $\tilde{x}_{k}=0$. For simplicity we take $k_{1}=0$ in Proposition 5. 
Proposition 6: Suppose that for $k \geqslant 0$ the system evolves on the sliding surface $\tilde{s}_{x, k}=0$, so that (neglecting terms in $h^{2}$ ) $\left|s_{x, k+1}\right| \leqslant h \varphi_{\max }+\left|\epsilon_{k}\right|$. Then,

$$
x_{k}=\left(1+h k_{x}\right)^{-1} x_{0}-h\left(1+h k_{x}\right)^{-1} \sum_{i=0}^{k-1} \zeta_{i, k} .
$$

with $\zeta_{i, k}=\left(1+h k_{x}\right)^{-i}\left(\epsilon_{k-1-i}+h^{2} \alpha_{k-1-i}+h \varphi_{k-i}\right)$.

Proof: From $s_{x, k+1}=0$ one easily derives

$x_{k+1}=\left(1+h k_{x}\right)^{-1} x_{k}-h\left(1+h k_{x}\right)^{-1}\left(\epsilon_{k}+h^{2} \alpha_{k}+h \varphi_{k+1}\right)$

from which (42) is deduced.

The disturbance is therefore attenuated by a factor $h^{2}$ on the state "position" $x_{k}$. Similarly to (13), using (1) we may rewrite the discrete-time closed-loop system as

$$
\left\{\begin{array}{l}
s_{x, k+1}=s_{x, k}-h k_{e} \dot{e}_{k}-h k_{s} s_{e, k}-h M_{v} \tau_{1, k+1} \\
\quad-h M_{x} \tau_{2, k+1}+h \varphi\left(x_{k+1}, t_{k+1}\right) \\
s_{e, k+1}=s_{e, k}-h k_{s} s_{e, k}-h M_{v} \tau_{1, k+1}+h \varphi\left(x_{k+1}, t_{k+1}\right) \\
x_{k+1}=x_{k}+h \dot{x}_{k+1} \\
e_{k+1}=e_{k}+h \dot{e}_{k+1} \\
\tau_{1, k+1}=\operatorname{proj}\left([-1,1] ; \frac{s_{e, k}-h k_{s} s_{e, k}}{h M_{v}}\right) \\
\tau_{2, k+1}=\operatorname{proj}\left([-1,1] ; \frac{s_{x, k}-h k_{e} \dot{e}_{k}-h k_{s} s_{e, k}-h M_{x} \tau_{1, k+1}}{h M_{x}}\right) .
\end{array}\right.
$$

One has also $s_{e, k}=\dot{e}_{k}+k_{e} e_{k}, s_{x, k}=\dot{x}_{k}+k_{x} x_{k}$, so that $x_{k+1}=\left(1+h k_{x}\right)^{-1}\left(x_{k}+h s_{x, k+1}\right)$ and $e_{k+1}=$ $\left(1+h k_{e}\right)^{-1}\left(e_{k}+h s_{e, k+1}\right)$. The controller has a nested-projection structure and is easily implementable at time $t=t_{k}$ with the knowledge of $x_{k}, x_{k-1}$ and $e_{k}, e_{k-1}$.

\section{B. Position Feedback Stabilization of a Double Integrator}

Let us now pass to other types of sliding-mode discontinuous controllers which have been proposed in the literature, known as the twisting and super-twisting algorithms [14], [35, $\S 3.6 .2,3.6 .3]$. They possess advantages (finite-time stability of the origin, better disturbance attenuation); however, their stability analysis is more intricate.

1) Finite-Time Stabilizing State Feedback Synthesis: To begin with, we present a static feedback controller that globally stabilizes the double integrator

$$
\dot{x}(t)=y(t), \quad \dot{y}(t)=u(t) .
$$

A feedback law $u(x, y)$ is further referred to as finite-time stabilizing if it renders the origin of the closed-loop system (45) a finite-time stable equilibrium as defined in [27]. The following state feedback

$$
u \in-\mu \operatorname{sgn}(y)-\nu \operatorname{sgn}(x)
$$

with parameters $\nu>\mu>0$ is proposed to globally stabilize the double integrator (45).
Theorem 1: Consider the dynamics of the closed-loop system in (45), (46). This dynamics has a unique fixed point $(x, y)=$ $(0,0)$ which is globally finite-time stable, provided that the controller parameters are such that $\nu>\mu>0$.

The proof may be found in the Example 3.2 and section 4.6 of [27]. Let us now consider the disturbance-corrupted version:

$$
\dot{x}(t)=y(t), \dot{y}(t)=u(t)+\varphi(x(t), y(t), t)
$$

and investigate the robustness properties of the closed-loop system (46), (47) against external disturbances $\varphi(x, y, t)$, being a locally integrable function on all potential trajectories $x(t), y(t)$. According to [27, Th. 4.2], the disturbed system in (46), (47) renders the system finite-time stable, regardless of whichever disturbance $\varphi(\cdot)$ with a uniform upper bound

$$
\operatorname{ess} \sup _{t \geq 0}|\varphi(x(t), y(t), t)| \leqslant \varphi_{\max }
$$

on its magnitude such that

$$
0<\varphi_{\max }<\mu<\nu-\varphi_{\max }
$$

affects the system. This robustness property is achieved due to the high frequency controller switching in the sliding mode of the second-order that occurs in the origin.

Theorem 2: [27, Sec. 4.6] Given $\mu$ and $\nu>0$, the closed-loop system in (46), (47) has a unique fixed point $\left(x^{*}, y^{*}\right)=(0,0)$ which is globally asymptotically finite-time stable, regardless of whichever disturbance $\varphi(\cdot)$, satisfying (48) and (49), affects the system.

Let us propose the following implicit Euler time-discretization, where $\varphi_{k+1} \triangleq \varphi\left(x_{k+1}, y_{k+1}, t_{k+1}\right)$ :

$$
\left\{\begin{array}{l}
\tilde{x}_{k+1}=x_{k}+h \tilde{y}_{k+1} \\
\tilde{y}_{k+1}=y_{k}-h \nu \tau_{1, k+1}-h \mu \tau_{2, k+1} \\
\tau_{1, k+1} \in \operatorname{sgn}\left(\tilde{x}_{k+1}\right) \\
\tau_{2, k+1} \in \operatorname{sgn}\left(\tilde{y}_{k+1}\right) \\
x_{k+1}=x_{k}+h y_{k+1} \\
y_{k+1}=y_{k}-h \nu \tau_{1, k+1}-h \mu \tau_{2, k+1}+h \varphi_{k+1}
\end{array}\right.
$$

from which it follows applying (1) to the second and the fourth lines of (50) that

$$
\tau_{2, k+1}=\operatorname{proj}\left([-1,1] ; \frac{y_{k}-h \nu \tau_{1, k+1}}{h \mu}\right) .
$$

The discrete-time system in (50) is still constructed along the same lines as the ones in the foregoing sections: one computes the input from a nominal unperturbed system (the first four lines of (50)) and then one injects the computed input into the plant dynamics (the last two lines of (50)). However this time there is no decoupling between the $\tilde{x}_{k}$-dynamics and the $\tilde{y}_{k}$-dynamics. It is easily checked that $\left(x^{*}, y^{*}\right)=(0,0)$ is the unique fixed point of the unperturbed system (50) [take $\varphi_{k+1}=0$ in (50)]. The next results hold. 
Lemma 3: The controller $\left(\tau_{1, k+1}, \tau_{2, k+1}\right)^{T}$ is a causal input at time $t=t_{k}$ and there is no singularity in $\tau_{1, k+1}$ as $h$ tends to zero.

The proof is in Appendix B.

Lemma 4: Suppose $\varphi_{k} \equiv 0$. a) Let $\left|y_{k_{0}}-h \nu \tau_{1, k_{0}+1}\right| \leqslant h \mu$ and $x_{k_{0}}=0$ for some $k_{0} \geqslant 0$. Then $\left|y_{k}-h \nu \tau_{1, k+1}\right| \leqslant h \mu$ for all $k \geqslant k_{0}$, so that $x_{k+1}=y_{k+1}=0$ for all $k \geqslant k_{0}$. b) If $x_{k_{0}}=y_{k_{0}}=0$ for some $k_{0} \geqslant 0$, then $x_{k}=y_{k}=0$ and $\left|y_{k}-h \nu \tau_{1, k+1}\right| \leqslant h \mu$ for all $k>k_{0}$.

The proof is in Appendix C. Lemma 4 says that (in the unperturbed case), once the system has reached the fixed point it stays on it without any spurious oscillations. This is an interesting property of implicit Euler schemes [1]. The lemma shows also that mode (iii) is the unperturbed system's mode at the equilibrium point. The following results characterize the disturbance attenuation on the nominal system sliding mode.

Proposition 7: Suppose that $\tilde{x}_{k+1}=0$ and $\tilde{y}_{k+1}=0$. Then $y_{k+1}=h \varphi_{k+1}$ and $x_{k+1}=h^{2} \varphi_{k+1}$. Therefore, $\left|y_{k+1}\right| \leqslant$ $h \varphi_{\max }$ and $\left|x_{k+1}\right| \leqslant h^{2} \varphi_{\max }$.

Proof: From the first line of (50) it follows that $x_{k}=0$. From the third and last lines one has $y_{k+1}=h \varphi_{k+1}$. From the fifth line it follows that $x_{k+1}=h^{2} \varphi_{k+1}$.

There is however a major difference between (50) and the systems in the foregoing sections. Indeed the conditions of Proposition 7 can hold only at one time step. Assume that $\tilde{x}_{k+1}=$ $\tilde{y}_{k+1}=0$. Then $x_{k+1}=h^{2} \varphi_{k+1}$ and $y_{k+1}=h \varphi_{k+1}$, so that $\tilde{x}_{k+2}=x_{k+1}+h \tilde{y}_{k+2}$ implies $\tilde{y}_{k+2}=-h \varphi_{k+1}$. One must refine Proposition 7.

Proposition 8: Suppose that $\tilde{x}_{k+1}=0$ for some $k \geqslant 0$. Then $x_{k+1}=h^{2} \varphi_{k+1}$. Moreover if $\tilde{x}_{k+1}=0$ for all $k \geqslant 0$, then $y_{k+1}=h\left(\varphi_{k+1}-\varphi_{k}\right)$ and $\sum_{k=1}^{n} y_{k}=h\left(\varphi_{n}-\varphi_{0}\right)$, while $\tilde{y}_{k+1}=-h \varphi_{k}$ for all $k \geqslant 0$.

Proof: Let $\tilde{x}_{k+1}=0$. Then $x_{k}=-h \tilde{y}_{k+1}$. Thus, $-h \tilde{y}_{k+1}=x_{k}=-h y_{k}+h^{2} \nu \tau_{1, k+1}+h^{2} \mu \tau_{2, k+1}$, so that $x_{k}+h y_{k}=h^{2} \nu \tau_{1, k+1}+h^{2} \mu \tau_{2, k+1}$. Thus, $x_{k+1}=$ $x_{k}+h y_{k}-h^{2} \nu \tau_{1, k+1}-h^{2} \mu \tau_{2, k+1}+h^{2} \varphi_{k+1}=h^{2} \varphi_{k+1}$. Now we have $\tilde{x}_{k+1}=0$ implies $x_{k+1}=h^{2} \varphi_{k+1}$ and $\tilde{x}_{k+2}=0$ implies $x_{k+2}=h^{2} \varphi_{k+2}$. Thus, $x_{k+2}=$ $x_{k+1}+h y_{k+1}=h^{2} \varphi_{k+1}+h y_{k+1}=h^{2} \varphi_{k+2}$ so that $y_{k+1}=h\left(\varphi_{k+2}-\varphi_{k+1}\right)$. The same can be done for the next step if $\tilde{x}_{k+3}=0$. The sum immediately follows. Finally, $y_{k+1}=h\left(\varphi_{k+1}-\varphi_{k}\right)=\tilde{y}_{k+1}+h \varphi_{k+1}$ so that $\tilde{y}_{k+1}=-h \varphi_{k}$.

Propositions 7 and 8 show that the disturbance attenuation holds for (50); however, the nominal system's trajectories cannot slide along both $\tilde{x}_{k}=0$ and $\tilde{y}_{k}=0$.

Remark 2: The differential inclusions in (3), (10), (35) and (46), (47) are written more compactly as

$$
\left\{\begin{array}{l}
\dot{z}(t)=A z(t)-B \tau(t)+\Phi(z(t), t) \\
\tau(t) \in \operatorname{Sgn}(C z(t))
\end{array}\right.
$$

with obvious definitions of $z, A, B, C$, and $\Phi(\cdot)$. The results in [1] do not apply to (10), (35) and (46), (47) because the "input-output" condition $P B=C^{T}$ with $P=P^{T}>0$ that is central in [1] is not satisfied for these systems. This means that the underlying maximal monotonicity arguments which allow one to draw conclusions about the convergence in [1], are absent in (10), (35) and (46), (47). The same applies to (47), (53), and (58). Finally, the twisting algorithm is more complex than (10) and (35) because it is the equilibrium that is reached in finite time, not a codimension one sliding surface that allows one to treat the problem as a two-stage problem.

2) Finite-Time Velocity Observer Design: The focus of the present study is on the stability analysis of the velocity observer of the supertwisting observer

$$
\left\{\begin{array}{l}
\dot{\hat{x}}(t)=\hat{y}(t)+k_{1}|x(t)-\hat{x}(t)|^{\frac{1}{2}} \operatorname{sgn}(x(t)-\hat{x}(t)) \\
\quad+k_{2}(x(t)-\hat{x}(t)) \\
\dot{\hat{y}}(t) \in u(t)+k_{3} \operatorname{sgn}(x(t)-\hat{x}(t))+k_{4}(x(t)-\hat{x}(t))
\end{array}\right.
$$

that was first proposed in [11] with $k_{2}, k_{4}=0$ and is now augmented with nontrivial linear gains $k_{2}, k_{4}>0$. Clearly, the observation error $e=\left(e_{1} e_{2}\right)^{T}, e_{1}=x-\hat{x}, e_{2}=y-\hat{y}$ between the state of the double integrator (45) and that of the velocity observer (53) proves to be governed by the following second-order system:

$$
\left\{\begin{array}{l}
\dot{e}_{1}(t) \in e_{2}(t)-k_{1} \sqrt{\left|e_{1}(t)\right|} \operatorname{sgn}\left(e_{1}(t)\right)-k_{2} e_{1}(t) \\
\dot{e}_{2}(t) \in-k_{3} \operatorname{sgn}\left(e_{1}(t)\right)-k_{4} e_{1}(t) .
\end{array}\right.
$$

The following result is extracted from [26] and [28].

Theorem 3: Given $k_{1}, k_{3}>0, k_{2}, k_{4} \geqslant 0$, the system (54) is globally finite-time stable.

In the rest of this section, we carry out the subordination for the observer gains $k_{i}, i=1,2,3,4$ that ensures the robustness of the perturbed dynamics:

$$
\left\{\begin{array}{l}
\dot{e}_{1}(t)=e_{2}(t)-k_{1} \sqrt{\left|e_{1}(t)\right|} \operatorname{sgn}\left(e_{1}(t)\right)-k_{2} e_{1}(t) \\
\dot{e}_{2}(t)=\varphi(x(t), y(t), t)-k_{3} \operatorname{sgn}\left(e_{1}(t)\right)-k_{4} e_{1}(t) .
\end{array}\right.
$$

As a matter of fact, this dynamics corresponds to the observation errors $e=\left(e_{1} e_{2}\right)^{T}, e_{1}=x-\hat{x}, e_{2}=y-\hat{y}$ between the state of the velocity observer (53) and that of the double integrator (47), affected by an admissible external disturbance.

Theorem 4: Let the system (55) be affected by a uniformly bounded disturbance (48). Furthermore, let the system gains be such that

$$
k_{1}, k_{3}>0, k_{2}, k_{4} \geqslant 0, \quad \text { if } k_{4}=0 \text { then } k_{2}=0 \text {. }
$$

Then the system (55) is globally finite-time stable whenever the upper bound $\varphi_{\max }$ on the magnitude of the external disturbance $\varphi(x, y, t)$ meets the condition

$$
\varphi_{\max }<\min \left\{\frac{k_{1}}{2}, \frac{k_{1} k_{3}}{1+k_{1}}\right\} .
$$

The proof of Theorem 4 follows the same line of reasoning as that proposed in [26] and [28] and it is therefore omitted.

3) Finite-Time Stabilizing Position Feedback Synthesis: In this section, we proceed with the design of the position feedback, stabilizing the double integrator in finite time. For this purpose, we substitute the velocity estimate $\hat{y}$ in the state feedback (46) for $y$ and, if desired, augment the resulting control law with the term $k_{3} \operatorname{sgn}\left(e_{1}\right)$ that compensates the disturbance $w$ on 
the sliding manifold $e_{1}=e_{2}=0$, to arrive at the finite-time stabilizing position feedback law:

$$
u \in-\mu \operatorname{sgn}(\hat{y})-\nu \operatorname{sgn}(x)
$$

(or at

$$
u \in-\mu \operatorname{sgn}(\hat{y})-\nu \operatorname{sgn}(x)-k_{3} \operatorname{sgn}\left(e_{1}\right)
$$

with the disturbance compensating term). Then the closed-loop system (47), (53), driven by (58) (or by (59), respectively) proves to be globally finite-time stable regardless of whichever admissible disturbance affects the system.

Theorem 5: Let the system (47) be affected by a uniformly bounded disturbance (48) and let it be driven by the observerbased dynamic feedback (53), (58) [respectively, (59)] with positive controller gains $\mu, \nu$ subject to (49), and with observer parameters $k_{i}, i=1,2,3,4$, satisfying conditions (56), (57). Then the closed-loop system (47), (53), and (58) is globally finite-time stable.

Proof: The closed-loop system (47), (53), and (58) rewritten in terms of the observation error (55), meets the conditions of Theorem 4. By applying Theorem 4 to the observation error system (55), we conclude that starting from a finite time instant $T$, the closed-loop system evolves on the manifold $e=0$ where $\hat{y}=y$, thereby ensuring that the position control signal (58) coincides with the state feedback signal (46). To complete the proof it remains to apply Theorem 2 to (47), (53), (58) for $t \geqslant T$ when the position feedback equals the state feedback. The global asymptotic stability of the closed-loop system (47), (53), and (58) is thus established.

The system in (47) and (58) and (53) is discretized as follows:

$$
\left\{\begin{array}{l}
\tilde{x}_{k+1}=x_{k}+h \tilde{y}_{k+1} \\
\tilde{y}_{k+1}=y_{k}-h \mu \tau_{2, k+1}-h \nu \tau_{1, k+1} \\
\hat{x}_{k+1} \in \hat{x}_{k}+h k_{1}\left|x_{k}-\hat{x}_{k}\right|^{\frac{1}{2}} \operatorname{sgn}\left(\tilde{x}_{k+1}-\hat{x}_{k+1}\right) \\
\quad+h k_{2}\left(\tilde{x}_{k+1}-\hat{x}_{k+1}\right)+h \hat{y}_{k+1} \\
\hat{y}_{k+1} \in \hat{y}_{k}-h \mu \tau_{2, k+1}-h \nu \tau_{1, k+1} \\
\quad+h k_{3} \operatorname{sgn}\left(\tilde{x}_{k+1}-\hat{x}_{k+1}\right)+h k_{4}\left(\tilde{x}_{k+1}-\hat{x}_{k+1}\right) \\
x_{k+1}=x_{k}+h y_{k+1} \\
y_{k+1}=y_{k}-h \mu \tau_{2, k+1}-h \nu \tau_{1, k+1}+h \varphi_{k+1} \\
\tau_{1, k+1} \in \operatorname{sgn}\left(\tilde{x}_{k+1}\right), \tau_{2, k+1} \in \operatorname{sgn}\left(\hat{y}_{k+1}\right)
\end{array}\right.
$$

Lemma 5: The unperturbed discrete-time multivalued system (60) possesses the unique equilibrium point $\left(\tilde{x}^{*}, \tilde{y}^{*}, \hat{x}^{*}, \hat{y}^{*}, x^{*}, y^{*}\right)^{T}=(0,0,0,0,0,0)^{T}$.

Proof: recall that in the unperturbed case we may consider that $\tilde{x}_{k}=x_{k}$ and $\tilde{y}_{k}=y_{k}$ for all $k$. From the first line of (60) it follows that $x^{*}=x^{*}+h y^{*} \Rightarrow y^{*}=0$. From the second line one has $y^{*}=y^{*}-h \mu \tau_{2}^{*}-h \nu \tau_{1}^{*}$, so that $\mu \tau_{2}^{*}+\nu \tau_{1}^{*} \ni 0 \Leftrightarrow$ $0 \in \operatorname{sgn}\left(x^{*}\right)+(\nu / \mu) \operatorname{sgn}\left(\hat{y}^{*}\right)$ which is satisfied if and only if $x^{*}=\hat{y}^{*}=0$ because $\nu>\mu$ from (49). From the third line $\hat{x}^{*}=$

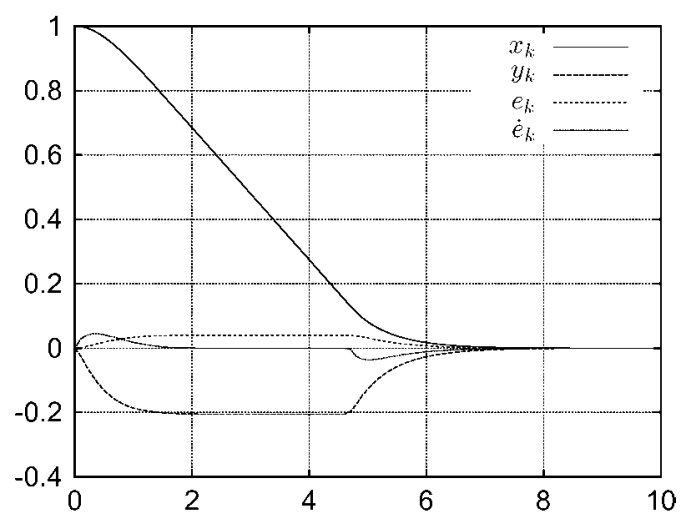

(a)

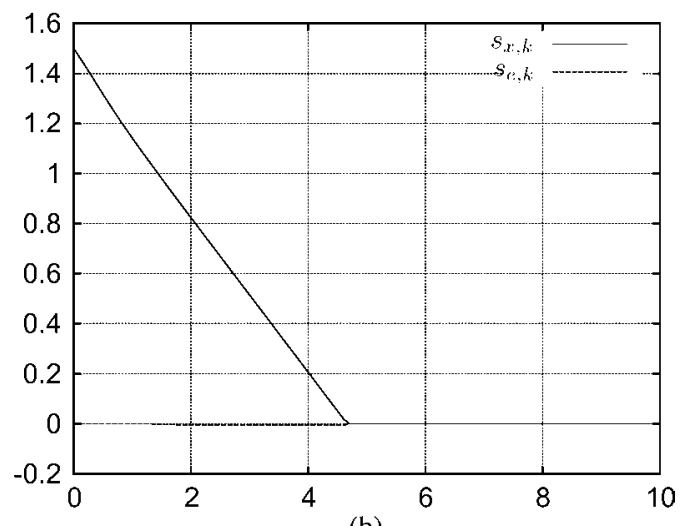

(b)

Fig. 4. Simulation of (44) with $\varphi(t)=0$. (a) State versus time. (b) Sliding variable $s(t)$

$\hat{x}^{*}+h k_{1} \sqrt{\left|x^{*}-\hat{x}^{*}\right|} \operatorname{sgn}\left(x^{*}-\hat{x}^{*}\right)+h k_{2}\left(x^{*}-\hat{x}^{*}\right)+h \hat{y}^{*}$ which is equivalent to $0 \in k_{1} \sqrt{\left|x^{*}-\hat{x}^{*}\right|} \operatorname{sgn}\left(x^{*}-\hat{x}^{*}\right)+k_{2}\left(x^{*}-\hat{x}^{*}\right)$. The unique solution of this generalized equation is $x^{*}=\hat{x}^{*}$, therefore $\hat{x}^{*}=0$.

Notice from the fourth and seventh lines of (60) that

$$
\tau_{2, k+1}=\operatorname{proj}\left([-1,1] ; \zeta_{k}\right)
$$

where (1) has been used and

$$
\zeta_{k}=\frac{\frac{\hat{y}_{k}}{h}-\nu \tau_{1, k+1}+k_{3} \operatorname{sgn}\left(\tilde{x}_{k+1}-\hat{x}_{k+1}\right)+k_{4}\left(\tilde{x}_{k+1}-\hat{x}_{k+1}\right)}{\mu} .
$$

Similarly to the twisting algorithm we may determine three main modes for (60):

- (i) if $\left(\hat{y}_{k} / h\right)-\nu \tau_{1, k+1}+k_{3} \operatorname{sgn}\left(\tilde{x}_{k+1}-\right.$ $\left.\hat{x}_{k+1}\right)+k_{4}\left(\tilde{x}_{k+1}-\hat{x}_{k+1}\right) \geqslant \mu: \tau_{2, k+1}=1$, $\tau_{1, k+1}=\operatorname{proj}\left([-1,1] ;\left(\left(y_{k}-h \mu+\left(x_{k} / h\right)\right) / h \nu\right)\right), \tilde{y}_{k+1}=$ $y_{k}-h \mu-h \nu \operatorname{proj}\left([-1,1] ;\left(\left(y_{k}-h \mu+\left(x_{k} / h\right)\right) / h \nu\right)\right)$;

- (ii) if $h\left(\hat{y}_{k} / h\right)-\nu \tau_{1, k+1}+k_{3} \operatorname{sgn}\left(\tilde{x}_{k+1}-\right.$ $\left.\hat{x}_{k+1}\right)+k_{4}\left(\tilde{x}_{k+1}-\hat{x}_{k+1}\right) \leqslant-\mu: \tau_{2, k+1}=-1$, $\tau_{1, k+1}=\operatorname{proj}\left([-1,1] ;\left(\left(y_{k}+h \mu+\left(x_{k} / h\right)\right) / h \nu\right)\right), \tilde{y}_{k+1}=$ $y_{k}+h \mu-h \nu \operatorname{proj}\left([-1,1] ;\left(\left(y_{k}+h \mu+\left(x_{k} / h\right)\right) / h \nu\right)\right)$;

- (iii) if $\mid\left(\hat{y}_{k} / h\right)-\nu \tau_{1, k+1}+k_{3} \operatorname{sgn}\left(\tilde{x}_{k+1}-\right.$ $\left.\hat{x}_{k+1}\right)+k_{4}\left(\tilde{x}_{k+1}-\hat{x}_{k+1} \mid<\mu\right.$ : then one obtains $\tau_{2, k+1} \quad=\left(\left(\hat{y}_{k} / h\right)-\nu \tau_{1, k+1}+\right.$ $\left.\left.k_{3} \operatorname{sgn}\left(\tilde{x}_{k+1}-\hat{x}_{k+1}\right)+k_{4}\left(\tilde{x}_{k+1}-\hat{x}_{k+1}\right)\right) / \mu\right)$; let $\alpha=\left(1+h^{2} k_{4}+h k_{2}\right) /\left(h^{2} k_{3}+h k_{1}\left|x_{k}-\hat{x}_{k+1}\right|^{1 / 2}\right)$ and $\beta=\left(x_{k}-\hat{x}_{k}+h y_{k}\right) /\left(h^{2} k_{3}+h k_{1}\left|x_{k}-\hat{x}_{k+1}\right|^{1 / 2}\right)$, 
then $\tilde{x}_{k+1}-\hat{x}_{k+1}=(1 / \alpha)(\beta-\operatorname{proj}([-1,1] ; \beta)$; finally $\tau_{1, k+1}=\operatorname{proj}\left([-1,1] ;\left(\left(x_{k}+h y_{k}-h \mu \tau_{2, k+1}\right) / h \nu\right)\right)$.

The decomposition into sub-modes becomes cumbersome and is not done here for the sake of paper's brevity. To provide an idea on how this works let us calculate $\hat{x}_{k+1}$ in a sub-mode of mode (i). Let us consider (i-1) such that $y_{k}-h \mu+\left(x_{k} / h\right)>$ $h \nu$. Then $\tau_{1, k+1}=1$. The condition for the activation of mode (i) thus boils down to $\left(\hat{y}_{k} / h\right)-\nu+k_{3} \operatorname{sgn}\left(\tilde{x}_{k+1}-\hat{x}_{k+1}\right)+$ $k_{4}\left(\tilde{x}_{k+1}-\hat{x}_{k+1}\right) \geqslant \mu$, which is equivalent to $k_{3} \operatorname{sgn}\left(\tilde{x}_{k+1}-\right.$ $\left.\hat{x}_{k+1}\right)+k_{4}\left(\tilde{x}_{k+1}-\hat{x}_{k+1}\right)>\mu+\nu-h \hat{y}_{k} \Leftrightarrow \tilde{x}_{k+1}-\hat{x}_{k+1}>$ $\max \left(0,\left(\left(\left(\hat{y}_{k} / h\right)+\mu+\nu-k_{3}\right) / k_{4}\right)\right)$. Then $\hat{x}_{k+1}=\hat{x}_{k}+$ $h k_{1}\left|x_{k}-\hat{x}_{k}\right|^{1 / 2}+h k_{2}\left(\tilde{x}_{k+1}-\hat{x}_{k+1}\right)+h \hat{y}_{k+1}$. Since $\tau_{2, k+1}=1$ we have $\tilde{x}_{k+1}=x_{k}+h\left(y_{k}-h \mu-h \nu\right)>0$ so that $\hat{x}_{k+1}=$ $\hat{x}_{k}+h k_{1}\left|x_{k}-\hat{x}_{k}\right|^{1 / 2}+h k_{2}\left(x_{k}+h y_{k}-h^{2} \mu-h^{2} \nu\right)-h k_{2} \hat{x}_{k+1}+$ $h \hat{y}_{k+1}$, and $\hat{y}_{k+1}=\hat{y}_{k}-h \mu-h \nu+h k_{3}+h k_{4}\left(\tilde{x}_{k+1}-\hat{x}_{k+1}\right)$. Hence,

$$
\begin{aligned}
\left(1+h k_{2}+h^{2} k_{4}\right) \hat{x}_{k+1}= & \hat{x}_{k}+h k_{1}\left|x_{k}-\hat{x}_{k}\right|^{\frac{1}{2}} \\
& +h\left(k_{2}+h k_{4}\right) x_{k} \\
& -h^{2}\left(h^{2} k_{4}+h k_{2}+1\right)(\mu+\nu) \\
& +h^{2}\left(k_{2}+h k_{4}\right) y_{k}+h \hat{y}_{k} .
\end{aligned}
$$

The computation of $\hat{y}_{k+1}$ is done in the same way, showing that the discrete-time observer dynamics [the third, fourth and seventh lines of (60)] is causal. Similar calculations may be done for the sub-modes (i-2): $y_{k}-h \mu+\left(x_{k} / h\right)<-h \nu$, (ii-1): $y_{k}+h \mu+\left(x_{k} / h\right)>h \nu,(\mathrm{ii}-2): y_{k}+h \mu+\left(x_{k} / h\right)<-h \nu$, and so on. Let us now prove that the disturbance attenuation holds on the nominal system sliding mode:

Lemma 6: Suppose that $\tilde{x}_{k+1}=0$, then $x_{k+1}=h^{2} \varphi_{k+1}$. If $\tilde{x}_{k+n}=0$ for all $n \geqslant 1$ then $y_{k+n+1}=h\left(\varphi_{k+n}-\varphi_{k+n-1}\right)$.

Proof: From (60) it follows that $\tilde{x}_{k+1}=0 \Rightarrow \tilde{y}_{k+1}=$ $-x_{k} / h$. Thus, $-\left(x_{k} / h\right)=y_{k}-h \mu \tau_{2, k+1}-h \nu \tau_{1, k+1}$, so that $y_{k+1}=y_{k}-\left(x_{k} / h\right)-y_{k}+h \varphi_{k+1}=-\left(x_{k} / h\right)+h \varphi_{k+1}$. Thus, $x_{k+1}=x_{k}-h\left(x_{k} / h\right)+h^{2} \varphi_{k+1}=h^{2} \varphi_{k+1}$. The second part of the lemma follows easily.

Remark 3 (Twisting Controllers Implementation): For both the twisting (Section III-B1) and the super-twisting (Section III-B3) controllers, we have shown that in all cases the inputs $\tau_{1, k+1}$ and $\tau_{2, k+1}$ can be computed from the knowledge of the state values at $t=t_{k}$ only. In practice, the controller

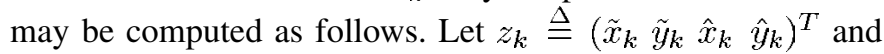
$\tau_{k} \triangleq\left(\tau_{1, k} \tau_{2, k}\right)^{T}$. Then the first four lines in (60) are rewritten compactly as

$$
\begin{gathered}
z_{k+1}=h F z_{k+1}+G z_{k}-h B \tau_{k+1}+h H\left(z_{k}\right) \operatorname{Sgn}\left(C z_{k+1}\right) \\
\mathbb{1} \\
z_{k+1}=\left(I_{4}-h F\right)^{-1}\left[G z_{k}-h B \tau_{k+1}+h H\left(z_{k}\right) \operatorname{Sgn}\left(C z_{k+1}\right)\right] \\
\mathbb{1} \\
z_{k+1}=\left(I_{4}-h F\right)^{-1}\left[G z_{k}+h\left(-B H\left(z_{k}\right)\right) \operatorname{Sgn}\left(D z_{k+1}\right)\right]
\end{gathered}
$$

where the matrices $F, G, H(\cdot), B, C, D$ can be easily identified from (60). The generalized equation (64) with unknown $z_{k+1}$ may be solved at each step using a specific iterative solver like those implemented in the software package SICONOS [1], [2], [4]. In the simulations of this paper Lemke's algorithm [10] has been used. The control algorithms presented in this paper can

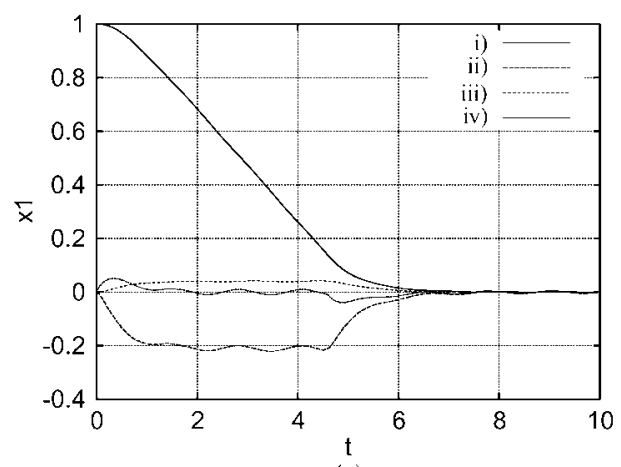

(a)

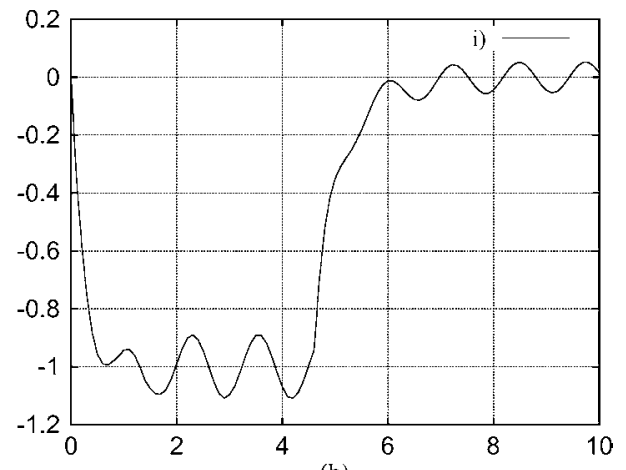

(b)

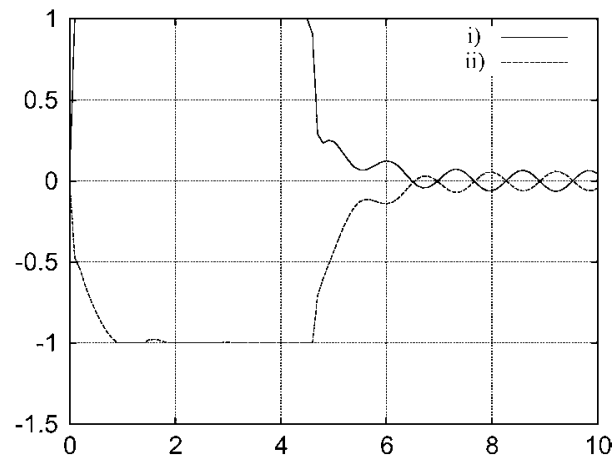

(c)

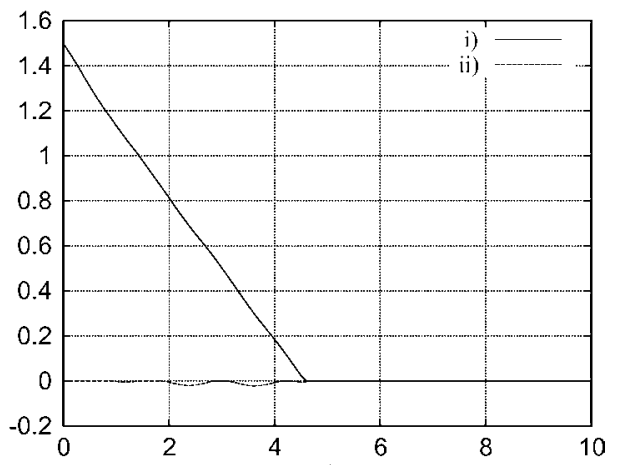

(d)

Fig. 5. Simulation of (44) with $\varphi(t)=\phi \sin (\omega t), \phi=0.1, \omega=5$. (a) State versus time (fine sampling): i) $x_{k}$, ii) $\dot{x}_{k}$, iii) $e_{k}$, iv) $\dot{e}_{k}$. (b) Control versus time i) $u_{k}$. (c) Multiplier versus time: i) $\tau_{1, k}$, ii) $\tau_{2, k}$. (d) Sliding variable i) $s_{x, k}$ ii) $s_{e, k}$.

therefore easily be implemented online. The same applies to (50).

\section{Numerical Simulations}

The system in (44) is simulated with $\varphi=\varphi(t)$ under the same conditions as those of Section II-D, with the software package 


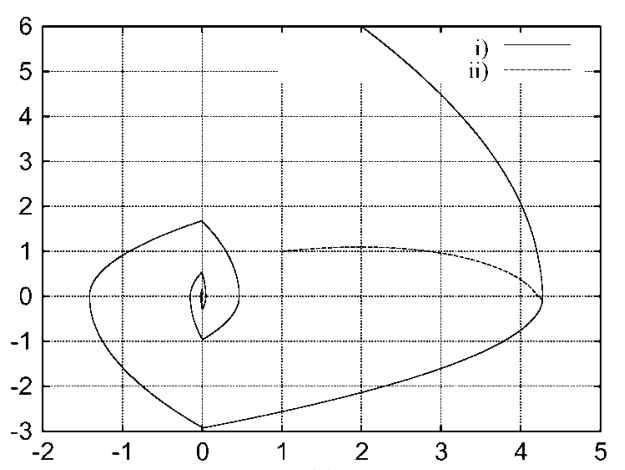

(a)
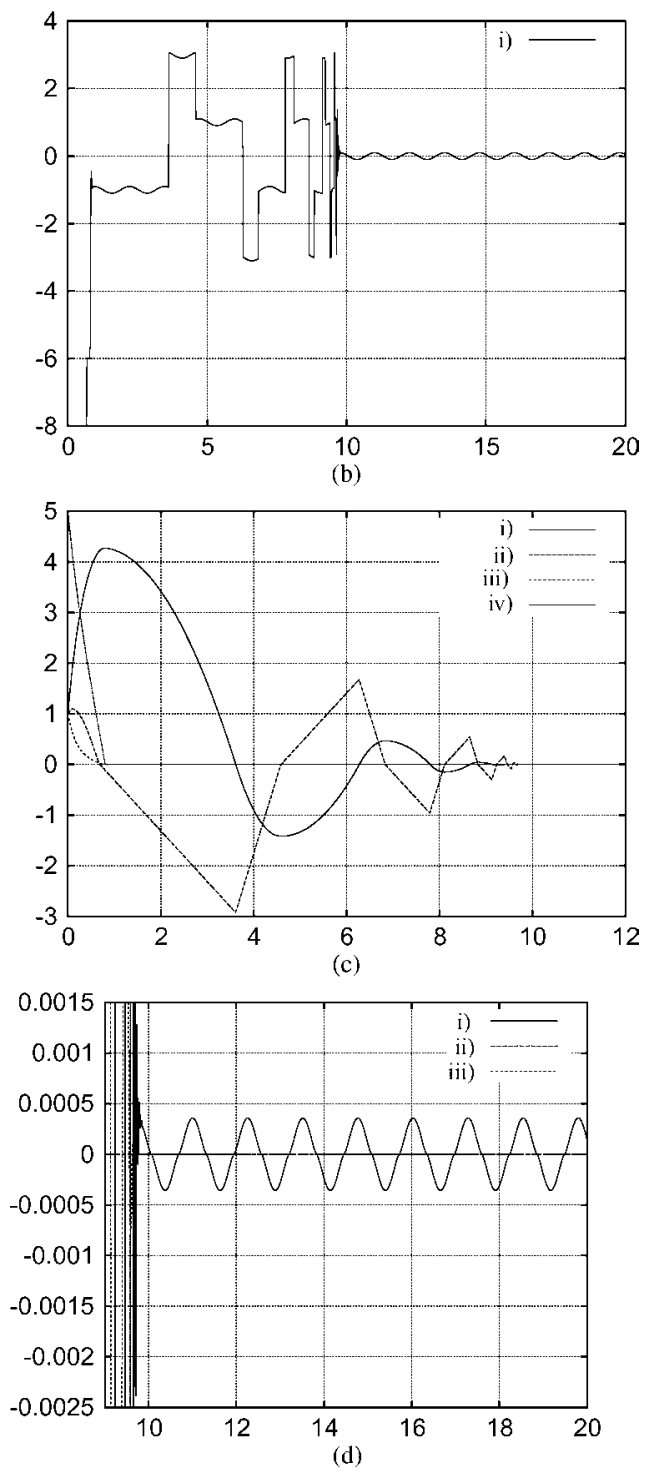

Fig. 6. Simulation of (60) with $\varphi(t)=\phi \sin (\omega t), \phi=0.1, \omega=5$. (a) Phase portrait. i) State $x_{k}$ versus $y_{k}$ ii) observer $\hat{x}_{k}$ versus $\hat{y}_{k}$; (b) control versus time i) $u_{k}$; (c) state and error versus time i) $\hat{x}_{k}$ ii) $\hat{y}_{k}$ iii) $e_{1, k}$ iv) $e_{2, k}$. (d) Sliding variable versus time. i) $\operatorname{sgn}\left(\hat{y}_{k}\right)$ ii) $\operatorname{sgn}\left(e_{1, k}\right)$ iii) $\operatorname{sgn}\left(\tilde{x}_{k}\right)$.

SICONOS. With $\varphi(t) \equiv 0$, the results are depicted on Fig. 4 . With $\varphi(t)=\phi \sin (\omega t) \phi=0.1, \omega=5$, the results are depicted on Fig. 5. We have chosen $k_{e}=k_{s}=5, k_{x}=1.5, M_{x}=1$, $M_{v}=2$. In Fig. 5(c) and 5(d), since $\tau_{1, k}$ and $\tau_{2, k}$ are inside $(-1 ; 1)$, one notices that the sliding surfaces $s_{e}=0$ and $s_{x}=0$ are reached in finite time as expected from Proposition 4.

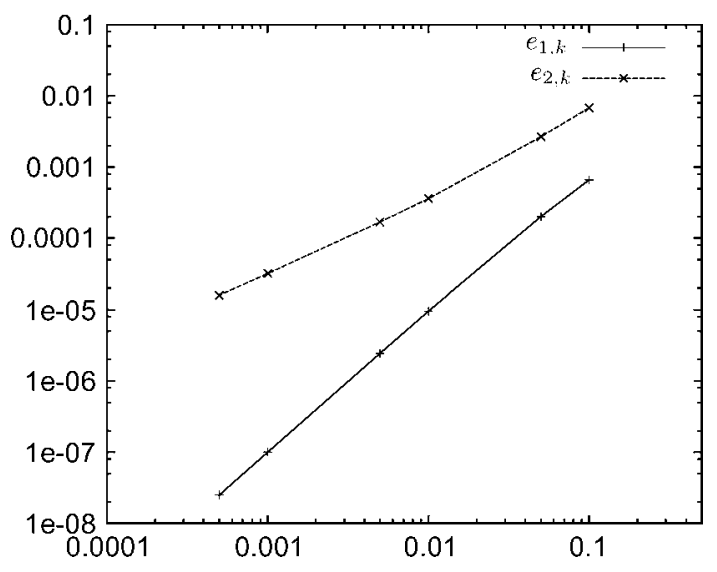

Fig. 7. Double logarithmic plot of the attenuation of the disturbance with respect to the sampling time.

The system in (60) is simulated with $\varphi=\varphi(t)$ with the same condition except for the sampling time chosen as $h=10^{-2}$. The initial conditions are $x_{0}=2, y_{0}=6, \hat{x}_{0}=1, \hat{y}_{0}=1$. We have chosen $\mu=1, \nu=2$ and $k_{1}=k_{2}=k_{3}=k_{4}=5$. With $\varphi(t)=\phi \sin (\omega t) \phi=0.1, \omega=5$, the results are depicted in Fig. 6. In Fig. 6(d), one notices again that the sliding surfaces are reached in finite time as expected in Theorem 5 . The attenuation is shown on Fig. 7 and we notice that $e_{1, k}=\mathcal{O}\left(h^{2}\right)$ and $e_{2, k}=\mathcal{O}(h)$. It is worth noting that the origin is attained after an infinite number of events (the switches of the sign functions) in the continuous-time twisting controllers. This can be seen in Fig. 6(b) and (d). Despite we have no convergence proof for the discrete-time solutions of the twisting algorithms, it is known that a very nice feature of backward Euler time-stepping methods is that they can handle accumulations of events (Zeno phenomena), see, e.g., [2, Ch. 1 and 10]. For this reason they are sometimes called event-capturing methods.

\section{CONCLUSION}

In this paper, a novel discrete-time implementation of slidingmode controllers is proposed. It is based on an implicit Euler method, and also applies to the zero-order-hold discretization. The controllers are simple and take the form of projections on the interval $[-1,1]$, or may be computed from simple quadratic programs. Most importantly the discrete-time controllers are able to represent the intrinsic multivalued feature of their continuous-time counterparts hence avoiding fast switches and highgain behaviors. The analysis shows that a smooth stabilization on the sliding surfaces is obtained in the case there is no disturbance (chattering-free controllers), while when a disturbance is present its effects are attenuated by factors $h$ or $h^{2}$. These properties are independent of the sampling period magnitude, which can be large. The controller has the nice property that the continuous-time and the discrete-time sliding surfaces are the same. Many simulation results illustrate the theory. Future works should concern the proof of convergence to the origin in a finite number of steps for the discrete-time twisting and super-twisting algorithms (as a complement to the numerical simulations presented in this paper), the extension towards other 
sliding-mode controllers (like systems with mismatched uncertainties), the numerical study of some optimal control problems that take the form of nonlinear variable-structure systems [8], and experimental comparisons with existing solutions for chattering reduction [6].

\section{APPENDIX A}

\section{PROOF OF PROPOSITION 2}

From (11) we have

$$
\left\{\begin{array}{l}
\tilde{e}_{k+1}=e_{k}-\alpha h \tau_{2, k+1} \\
\tau_{2, k+1} \in \operatorname{sgn}\left(\tilde{e}_{k+1}\right)
\end{array}\right.
$$

which is exactly the first two lines in (4). Therefore, the conclusions drawn for (4) apply, just replacing $a$ by $\alpha$. Thus, the $e_{k}$-dynamics is $e_{k+1}=e_{k}-\alpha h \operatorname{proj}\left([-1,1] ;\left(e_{k} / \alpha h\right)\right)+h \varphi_{k+1}$. After $k_{0}$ the discrete trajectory evolves on the sliding surface $\tilde{e}_{k}=0$ while $\tau_{2, k+1}=e_{k} / \alpha h$ and $e_{k}=h \varphi_{k+1}$, and one obtains using (1):

$$
\begin{aligned}
& \left\{\begin{array}{l}
\tilde{x}_{k+1}=x_{k}-h \varphi_{k+1}-a h \tau_{1, k+1} \\
\tau_{1, k+1} \in \operatorname{sgn}\left(\tilde{x}_{k+1}\right) \\
x_{k+1}=x_{k}-a h \tau_{1, k+1}
\end{array}\right. \\
& \Leftrightarrow \quad x_{k+1}=x_{k}-a h \operatorname{proj}\left([-1,1] ; \frac{x_{k}-h \varphi_{k+1}}{a h}\right) .
\end{aligned}
$$

Then we can redo the same calculations as in the proof of Proposition 1 (by replacing $x_{k}$ by $x_{k}-h \varphi_{k+1}$ in the first line of (4), and $x_{k}+h \varphi_{k+1}$ by $x_{k}$ in the third line), to infer that after a finite number of steps one gets $\tilde{x}_{k}=0, \tau_{1, k+1}=\left(x_{k}-\right.$ $\left.h \varphi_{k+1}\right) / a h$, and

$$
x_{k+1}=x_{k}-e_{k}-\left(x_{k}-h \varphi_{k+1}\right)+h \varphi_{k+1}=h \varphi_{k+1} .
$$

Indeed let us now assume that

$$
\left\{\begin{array}{l}
\tilde{x}_{k+1}=x_{k}-a h \tau_{1, k+1}-\alpha h \tau_{2, k+1}=0 \\
\tilde{e}_{k+1}=e_{k}-\alpha h \tau_{2, k+1}=0
\end{array}\right.
$$

that is

$$
\left\{\begin{array}{l}
\tau_{1, k+1}=\frac{x_{k}-e_{k}}{a h} \\
\tau_{2, k+1}=\frac{e_{k}}{\alpha h} .
\end{array}\right.
$$

After the update procedure (12), we get

$$
\left\{\begin{array}{l}
x_{k+1}=h \varphi_{k+1} \\
e_{k+1}=h \varphi_{k+1}
\end{array} .\right.
$$

We can conclude that once the sliding mode in $\tilde{x}$ and $\tilde{e}$ is reached we have

$$
\left\{\begin{array}{l}
x_{k+1}=h \varphi_{k+1} \\
e_{k+1}=h \varphi_{k+1}
\end{array}, \quad \text { for all } k \geqslant k_{0}\right.
$$

and

$$
\left\{\begin{array}{l}
\tau_{1, k+1}=0 \\
\tau_{2, k+1}=\frac{\varphi_{k}}{\alpha}, \quad \text { for all } k \geqslant k_{0}+1 .
\end{array}\right.
$$

\section{APPENDIX B}

PROOF OF LEMMA 3

One has $\tilde{y}_{k+1}=y_{k}-h \nu \tau_{1, k+1}-h \mu \operatorname{proj}\left([-1,1] ;\left(\left(y_{k}-\right.\right.\right.$ $\left.\left.\left.h \nu \tau_{1, k+1}\right) / h \mu\right)\right)$. Therefore, $\tilde{y}_{k+1}=y_{k}-h \nu \tau_{1, k+1}-$ $\operatorname{proj}\left([-h \mu, h \mu] ; y_{k}-h \nu \tau_{1, k+1}\right)$. Thus three "modes" are possible:

- (i) if $y_{k}-h \nu \tau_{1, k+1}>h \mu$ one gets $\tilde{y}_{k+1}=$ $y_{k}-h \nu \tau_{1, k+1}-h \mu, y_{k+1}=y_{k}-h \nu \tau_{1, k+1}-h \mu+h \varphi_{k+1}$ and $\tilde{x}_{k+1}=x_{k}+h\left(y_{k}-h \nu \tau_{1, k+1}\right)-h^{2} \mu$, $x_{k+1}=x_{k}+h\left(y_{k}-h \nu \tau_{1, k+1}\right)-h^{2} \mu+h^{2} \varphi_{k+1}$. Also $\tau_{1, k+1}=\left(1 / h^{2} \nu\right) \operatorname{proj}\left(\left[-h^{2} \nu, h^{2} \nu\right] ; x_{k}+h y_{k}-h^{2} \mu\right)$, $\tau_{2, k+1}=1$.

There are three sub-modes:

-(i-1) let $x_{k}+h y_{k}-h^{2} \mu>h^{2} \nu$ : then $\tau_{1, k+1}=1$, $y_{k}>h(\nu+\mu), x_{k+1}=x_{k}+h y_{k}-h^{2}(\nu+\mu)+h^{2} \varphi_{k+1}$, $y_{k+1}=y_{k}-h\left(\nu+\mu+\varphi_{k+1}\right)$.

- (i-2) let $x_{k}+h y_{k}-h^{2} \mu<-h^{2} \nu$ : then $\tau_{1, k+1}=-1$, $y_{k}>h(\mu-\nu), x_{k+1}=x_{k}+h y_{k}+h^{2}(\nu-\mu)+h^{2} \varphi_{k+1}$, $y_{k+1}=y_{k}+h\left(\nu-\mu+\varphi_{k+1}\right)$.

- (i-3) let $\left|x_{k}+h y_{k}-h^{2} \mu\right| \leqslant h^{2} \nu$ : then $x_{k}<0, \tau_{1, k+1}=$ $\left(x_{k}+h y_{k}-h^{2} \mu\right) / h^{2} \nu, x_{k+1}=h^{2} \varphi_{k+1}, y_{k+1}=$ $-\left(x_{k} / h\right)+h \varphi_{k+1}, \tilde{x}_{k+1}=0, \tilde{y}_{k+1}=-\left(x_{k} / h\right)$.

- (ii) if $y_{k}-h \nu \tau_{1, k+1}<-h \mu$ one gets $\tilde{y}_{k+1}=y_{k}-$ $h \nu \tau_{1, k+1}+h \mu, y_{k+1}=y_{k}-h \nu \tau_{1, k+1}+h \mu+h \varphi_{k+1}$ and $\tilde{x}_{k+1}=x_{k}+h\left(y_{k}-h \nu \tau_{1, k+1}\right)-h^{2} \mu, x_{k+1}=$ $x_{k}+h\left(y_{k}-h \nu \tau_{1, k+1}\right)+h^{2} \mu+h^{2} \varphi_{k+1}$. Also $\tau_{1, k+1}=$ $\left(1 / h^{2} \nu\right) \operatorname{proj}\left(\left[-h^{2} \nu, h^{2} \nu\right] ; x_{k}+h y_{k}+h^{2} \mu\right), \tau_{2, k+1}=-1$.

There are three sub-modes:

- (ii-1) let $x_{k}+h y_{k}+h^{2} \mu>h^{2} \nu$ : then $\tau_{1, k+1}=1$, $y_{k}<h(\nu-\mu), x_{k+1}=x_{k}+h y_{k}-h^{2}\left(\nu-\mu-\varphi_{k+1}\right.$, $y_{k+1}=y_{k}+h\left(\nu-\mu-\varphi_{k+1}\right)$.

- (ii-2) let $x_{k}+h y_{k}+h^{2} \mu<-h^{2} \nu$ : then $\tau_{1, k+1}=-1$, $y_{k}<-h(\nu+\mu), x_{k+1}=x_{k}+h y_{k}+h^{2}\left(\nu+\mu+\varphi_{k+1}\right.$, $y_{k+1}=y_{k}+h\left(\nu+\mu+\varphi_{k+1}\right)$.

- (ii-3) let $\left|x_{k}+h y_{k}+h^{2} \mu\right| \leqslant h^{2} \mu$ : then $\tau_{1, k+1}=$ $\left(x_{k}+h y_{k}+h^{2} \mu\right) / h^{2} \nu, x_{k+1}=h^{2} \varphi_{k+1}, \tilde{x}_{k+1}=0$, $y_{k+1}=-\left(x_{k} / h\right)+h \varphi_{k+1}, \tilde{y}_{k+1}=-\left(x_{k} / h\right)$.

- (iii) if $\left|y_{k}-h \nu \tau_{1, k+1}\right| \leqslant h \mu$ one gets $\tilde{y}_{k+1}=y_{k}-$ $h \nu \tau_{1, k+1}-\left(y_{k}-h \nu \tau_{1, k+1}\right)=0, y_{k+1}=h \varphi_{k+1}$ and $x_{k+1}=x_{k}+h^{2} \varphi_{k+1}$. Also $\tau_{1, k+1} \in \operatorname{sgn}\left(x_{k}\right), \tau_{2, k+1}=$ $\left(y_{k}-h \nu \tau_{1, k+1}\right) / h \mu$.

In (i) and (ii), the value for $\tau_{1, k+1}$ is obtained from the generalized equation $\tau_{1, k+1} \in \operatorname{sgn}\left(x_{k}+h y_{k}-h^{2} \nu \tau_{1, k+1}-h^{2} \mu\right)$ and using (1). In all cases $\tau_{2, k+1}$ is obtained from (51).

\section{APPENDIX C \\ PROOF OF LEMMA 4}

(a) From (iii) above it follows that $\left|y_{k_{0}}-h \nu \tau_{1, k_{0}+1}\right| \leqslant h \mu$ and $x_{k_{0}}=0$ imply $y_{k_{0}+1}=x_{k_{0}+1}=0$. Therefore, $\left|y_{k_{0}+1}-h \nu \tau_{1, k_{0}+2}\right|=h \nu\left|\tau_{1, k_{0}+2}\right|$. Now suppose that $h \nu\left|\tau_{1, k_{0}+2}\right|>h \mu$, one obtains from (i) and (ii) that $\tau_{1, k_{0}+2}=\left(1 / h^{2} \nu\right) \operatorname{proj}\left(\left[-h^{2} \nu, h^{2} \nu\right] ; x_{k_{0}+1}+h y_{k_{0}+1} \pm\right.$ $\left.h^{2} \mu\right)=\left(1 / h^{2} \nu\right) \operatorname{proj}\left(\left[-h^{2} \nu, h^{2} \nu\right] ; \pm h^{2} \mu\right)=\mu / \nu$ since $\mu<\nu$. So indeed $\left|y_{k_{0}+1}-h \nu \tau_{1, k_{0}+2}\right|=h \mu$, a contradiction. It follows that $h \nu\left|\tau_{1, k_{0}+2}\right| \leqslant h \mu$ and therefore $\left|y_{k_{0}+1}-h \nu \tau_{1, k_{0}+2}\right| \leqslant h \mu$ and $x_{k_{0}+2}=y_{k_{0}+2}=0$. Now $x_{k_{0}+2}=x_{k_{0}+1}=0$. We can repeat the reasoning at the next step and (a) is proved. (b) From $x_{k}=y_{k}=0$ we deduce that $x_{k+1}=h y_{k+1}$ and $y_{k+1}=-h \nu \tau_{1, k+1}-h \mu \tau_{2, k+1}$. Also $\tau_{1, k+1} \in \operatorname{sgn}\left(-h \nu \tau_{1, k+1}-h \mu \tau_{2, k+1}\right)$ form which we infer that $\tau_{1, k+1}=\operatorname{proj}\left([-1,1] ;-(\mu / \nu) \tau_{2, k+1}\right)$ while 
$\tau_{2, k+1}=\operatorname{proj}\left([-1,1] ;-(\nu / \mu) \tau_{1, k+1}\right)$. Since $\mu<\nu$ it follows that $\tau_{1, k+1}=-(\mu / \nu) \tau_{2, k+1}$. Also $\left|(\nu / \mu) \tau_{1, k+1}\right| \leqslant 1$ so that $\left|\tau_{1, k+1}\right| \leqslant(\mu / \nu)<1$. Hence, since $\tau_{1, k+1} \in \operatorname{sgn}\left(x_{k+1}\right)$, $x_{k+1}=0$. From the fact that $x_{k+1}=h y_{k+1}$ it follows that $y_{k+1}=0$. The reasoning can be repeated at the next step. Furthermore, it easily follows that $\left|\tau_{1, k+1}\right| \leqslant(\mu / \nu)$ so part (b) is proved.

\section{REFERENCES}

[1] V. Acary and B. Brogliato, "Implicit Euler numerical scheme and chattering-free implementation of sliding mode systems," Syst. Control Lett., vol. 59, pp. 284-293, 2010.

[2] V. Acary and B. Brogliato, Numerical Methods for Nonsmooth Dynamical Systems. $\quad$ New York: Springer, 2008, vol. 35, Lecture Notes in Applied and Computational Mechanics.

[3] V. Acary, O. Bonnefon, and B. Brogliato, Nonsmooth Modeling and Simulation for Switched Circuits. New York: Springer, 2011, vol. 69, Lecture Notes in Electrical Engineering.

[4] V. Acary and F. Pérignon, "An Introduction to SICONOS," INRIA Tech. Rep. RR-0340, Jul. 2007 [Online]. Available: http://hal.inria.fr/inria00162911/fr/

[5] G. Bartolini, A. Ferrara, and V. I. Utkin, "Adaptive sliding mode control in discrete-time systems," Automatica, vol. 31, no. 5, pp. 769-773, 1995.

[6] D. Biel and E. Fossas, "Some experiments on chattering suppression in power converters," in Proc. 18th IEEE Int. Conf. Control Applicat., Part of 2009 IEEE Multi-Conf. Syst. Control, Saint Petersburg, Russia, Jul. 8-10, 2009, pp. 1523-1528.

[7] J. Bastien and M. Schatzman, "Numerical precision for differential inclusions with uniqueness," ESAIM M2AN: Math. Model. Numer. Anal., vol. 36, no. 3, pp. 427-460, 2002.

[8] F. L. Chernous'ko, I. M. Ananievski, and S. A. Reshmin, Control of Nonlinear Dynamical Systems, ser. CCE series. London, U.K.: Springer-Verlag, 2008.

[9] J. Cortes, "Discontinuous dynamical systems. A tutorial on solutions, nonsmooth analysis, and stability," IEEE Control Syst., 28, no. 3, pp. 36-73, Jun. 2008

[10] R. W. Cottle, J. Pang, and R. E. Stone, The Linear Complementarity Problem. Boston, MA: Academic, 1992.

[11] J. Davila, L. Fridman, and A. Levant, "Second-order sliding mode observer for mechanical systems," IEEE Trans. Autom. Control, vol. 50, no. 11, pp. 1785-1789, Nov. 2005.

[12] S. V. Drakunov and V. I. Utkin, "On discrete-time sliding modes," in Proc. IFAC Nonlinear Control Syst. Design NOLCOS, Capri, Italy, 1989, pp. 273-278.

[13] F. Facchinei and J. S. Pang, Finite-Dimensional Variational Inequalities and Complementarity Problems. New York: Springer-Verlag, 2003, vol. 1, Series in Operations Research.

[14] L. Fridman and A. Levant, "Higher order sliding modes as a natural phenomenon in control theory," in Robust Control via Variable Structure and Lyapunov Techniques, F. Garafalo and L. Glielmo, Eds. London, U.K.: Springer-Verlag, 1996, vol. 217, Lecture Notes in Control and Information Science, pp. 107-133.

[15] K. Furuta, "Sliding mode control of a discrete system," Syst. Control Lett., vol. 14, pp. 145-152, 1990.

[16] Z. Galias and X. Yu, "Complex discretization behaviours of a simple sliding-mode control system," IEEE Trans. Circuits Syst.-II: Exp. Briefs, vol. 53, no. 8, pp. 652-656, Aug. 2006.

[17] Z. Galias and X. Yu, "Analysis of zero-order holder discretization of two-dimensional sliding-mode control systems," IEEE Trans. Circuits Syst.-II: Exp. Briefs, vol. 55, no. 12, pp. 1269-1273, Dec. 2008.

[18] IEEE Trans. Ind. Electron., vol. 55, 56, no. 11, 9, 2008, 2009, special issues (O. Kaynak, A. Bartoszewicz and V.I. Utkin, Guest Editors).

[19] H. Lee and V. I. Utkin, "Chattering analysis," in Advances in Variable Structure, C. Edwards, Ed. et al. New York: Springer, 2006, vol. 334, LNCIS, pp. 107-121.

[20] A. J. Koshkouei and A. S. I. Zinober, "Sliding mode control of discrete-time systems," ASME J. Dyn. Syst., Meas. Control, vol. 122, pp. 793-802, Dec. 2000.
[21] H. Lee and V. I. Utkin, "Chattering suppression methods in sliding mode control systems," Аnnu. Rev. Control, vol. 31, pp. 179-188, 2007.

[22] A. Levant, "Sliding order and sliding accuracy in sliding mode control," Int. J. Control, vol. 58, no. 6, pp. 1247-1263, 1993.

[23] A. Levant, "Chattering analysis," IEEE Trans. Autom. Control, vol. 55, no. 6, pp. 1380-1389, Jun. 2010.

[24] Modern Sliding Mode Control Theory. New Perspectives and Applications, G. Bartolini, L. Fridman, A. Pisano, and E. Usai, Eds. New York: Springer-Verlag, 2008, vol. 375, Lecture Notes in Control and Information Sciences.

[25] C. Milosavljevic, "General conditions for the existence of a quasi-sliding mode on the switching hyperplane in discrete variable structure systems," Autom. Remote Control, vol. 46, no. 3, pt. 1, pp. 307-314, Mar. 1985.

[26] J. Moreno and M. Osorio, "A Lyapunov approach to second-order sliding mode controllers and observers," in Proc. 47th IEEE Conf. Decision Control, Cancun, Dec. 9-11, 2008, pp. 2856-2861.

[27] Y. Orlov, Discontinuous Systems-Lyapunov Analysis and Robust Synthesis Under Uncertainty Conditions, ser. Communications and Control Engineering Series. Berlin, Germany: Springer-Verlag, 2009.

[28] Y. Orlov, Y. Aoustin, and C. Chevallereau, "Finite time stabilization of a double integrator-Part 1: Continuous sliding mode-based position feedback synthesis," IEEE Trans. Autom. Control, vol. 56, no. 3, pp. 614-618, Mar. 2011

[29] R. Ramos, D. Biel, E. Fossas, and F. Guinjoan, "Interleaving quasi-sliding-mode control of parallel-connected buck-based inverters," IEEE Trans. Ind. Electron., vol. 55, no. 11, pp. 3865-3873, Nov. 2008

[30] R. R. Ramos, D. Biel, E. Fossas, and F. Guinjoan, "A fixed-frequency quasi-sliding control algorithm: Application to power inverters design by means of FPGA implementation," IEEE Trans. Power Electron., vol. 18, no. 1, pp. 344-355, Jan. 2003.

[31] R. T. Rockafellar and R. J. B. Wets, Variational Analysis. New York: Springer-Verlag, 1998, vol. 317, Grundlehren der Mathematischen Wissenschaten.

[32] J. J. Slotine and W. Li, Applied Nonlinear Control. London, U.K.: Prentice-Hall, 1991.

[33] G. V. Smirnov, Introduction to the Theory of Differential Inclusions. Providence, RI: American Mathematical Society, 2002, vol. 41, Graduate Studies in Mathematics.

[34] V. I. Utkin, Sliding Modes in Control Optimization. Berlin, Germany: Springer-Verlag, 1992.

[35] V. Utkin, J. Guldner, and J. Shi, Sliding Mode Control in Electro-Mechanical Systems, ser. Automation and Control Engineering Series, 2nd ed. Boca Raton, FL: CRC, 2009.

[36] K. D. Young, V. I. Utkin, and U. Ozguner, "A control engineer's guide to sliding mode control," IEEE Trans. Control Syst. Technol., vol. 7, no. 3, pp. 328-342, May 1999.

[37] X. Yu, B. Wang, Z. Galias, and G. Chen, "Discretization effect on equivalent control-based multi-input sliding-mode control systems," IEEE Trans. Autom. Control, vol. 53, no. 6, pp. 1563-1569, Jul. 2008.

[38] F. Zhao and V. I. Utkin, "Adaptive simulation and control of variablestructure control systems in sliding regimes," Automatica, vol. 32, no. 7, pp. 1037-1042, 1996.

[39] B. Wang, X. Yu, and G. Chen, "ZOH discretization effect on singleinput sliding mode control systems with matched uncertainties," Automatica, vol. 45, pp. 118-125, 2009. 